\title{
Aptamer-Based Multiplexed Proteomic Technology for Biomarker Discovery
}

\author{
Larry Gold ${ }^{1,2 *}$, Deborah Ayers ${ }^{1}$, Jennifer Bertino ${ }^{1}$, Christopher Bock ${ }^{1}$, Ashley Bock ${ }^{1}$, Edward N. Brody ${ }^{1}$, \\ Jeff Carter ${ }^{1}$, Andrew B. Dalby ${ }^{1}$, Bruce E. Eaton ${ }^{4}$, Tim Fitzwater ${ }^{1}$, Dylan Flather ${ }^{1}$, Ashley Forbes ${ }^{1}$, Trudi \\ Foreman ${ }^{1}$, Cate Fowler ${ }^{1}$, Bharat Gawande ${ }^{1}$, Meredith Goss ${ }^{1}$, Magda Gunn ${ }^{1}$, Shashi Gupta ${ }^{1}$, Dennis \\ Halladay ${ }^{1}$, Jim Heil ${ }^{1}$, Joe Heilig ${ }^{1}$, Brian Hicke ${ }^{1}$, Gregory Husar ${ }^{1}$, Nebojsa Janjic ${ }^{1}$, Thale Jarvis ${ }^{1}$, Susan \\ Jennings ${ }^{1}$, Evaldas Katilius ${ }^{1}$, Tracy R. Keeney ${ }^{1}$, Nancy Kim ${ }^{1}$, Tad H. Koch ${ }^{4}$, Stephan Kraemer ${ }^{1}$, Luke \\ Kroiss $^{1}$, Ngan Le ${ }^{1}$, Daniel Levine ${ }^{3}$, Wes Lindsey ${ }^{1}$, Bridget Lollo ${ }^{1}$, Wes Mayfield ${ }^{1}$, Mike Mehan ${ }^{1}$, Robert \\ Mehler ${ }^{1}$, Sally K. Nelson ${ }^{1}$, Michele Nelson ${ }^{1}$, Dan Nieuwlandt ${ }^{1}$, Malti Nikrad ${ }^{1}$, Urs Ochsner $^{1}$, Rachel M. \\ Ostroff ${ }^{1}$, Matt Otis ${ }^{1}$, Thomas Parker ${ }^{3}$, Steve Pietrasiewicz ${ }^{1}$, Daniel I. Resnicow ${ }^{1}$, John Rohloff ${ }^{1}$, Glenn \\ Sanders ${ }^{1}$, Sarah Sattin ${ }^{1}$, Daniel Schneider ${ }^{1}$, Britta Singer ${ }^{1}$, Martin Stanton ${ }^{1}$, Alana Sterkel ${ }^{1}$, Alex \\ Stewart ${ }^{1}$, Suzanne Stratford ${ }^{1}$, Jonathan D. Vaught ${ }^{1}$, Mike Vrkljan ${ }^{1}$, Jeffrey J. Walker ${ }^{1 *}$, Mike Watrobka ${ }^{1}$, \\ Sheela Waugh ${ }^{1}$, Allison Weiss' ${ }^{1}$, Sheri K. Wilcox ${ }^{1}$, Alexey Wolfson ${ }^{1}$, Steven K. Wolk ${ }^{1}$, Chi Zhang ${ }^{1}$, Dom \\ Zichi $^{1}$
}

1 SomaLogic, Boulder, Colorado, United States of America, 2 Department of Molecular, Cellular, and Developmental Biology, University of Colorado, Boulder, Colorado, United States of America, 3 The Rogosin Institute and the Weill Medical College of Cornell University, New York, New York, United States of America, 4 Department of Chemistry and Biochemistry, University of Colorado, Boulder, Colorado, United States of America

\begin{abstract}
Background: The interrogation of proteomes ("proteomics") in a highly multiplexed and efficient manner remains a coveted and challenging goal in biology and medicine.

Methodology/Principal Findings: We present a new aptamer-based proteomic technology for biomarker discovery capable of simultaneously measuring thousands of proteins from small sample volumes (15 $\mu \mathrm{L}$ of serum or plasma). Our current assay measures 813 proteins with low limits of detection (1 pM median), 7 logs of overall dynamic range $(\sim 100 \mathrm{fM}-1 \mu \mathrm{M})$, and $5 \%$ median coefficient of variation. This technology is enabled by a new generation of aptamers that contain chemically modified nucleotides, which greatly expand the physicochemical diversity of the large randomized nucleic acid libraries from which the aptamers are selected. Proteins in complex matrices such as plasma are measured with a process that transforms a signature of protein concentrations into a corresponding signature of DNA aptamer concentrations, which is quantified on a DNA microarray. Our assay takes advantage of the dual nature of aptamers as both folded protein-binding entities with defined shapes and unique nucleotide sequences recognizable by specific hybridization probes. To demonstrate the utility of our proteomics biomarker discovery technology, we applied it to a clinical study of chronic kidney disease (CKD). We identified two well known CKD biomarkers as well as an additional 58 potential CKD biomarkers. These results demonstrate the potential utility of our technology to rapidly discover unique protein signatures characteristic of various disease states.
\end{abstract}

Conclusions/Significance: We describe a versatile and powerful tool that allows large-scale comparison of proteome profiles among discrete populations. This unbiased and highly multiplexed search engine will enable the discovery of novel biomarkers in a manner that is unencumbered by our incomplete knowledge of biology, thereby helping to advance the next generation of evidence-based medicine.

Citation: Gold L, Ayers D, Bertino J, Bock C, Bock A, et al. (2010) Aptamer-Based Multiplexed Proteomic Technology for Biomarker Discovery. PLoS ONE 5(12): e15004. doi:10.1371/journal.pone.0015004

Editor: Fabrizio Gelain, University of Milan-Bicocca, Italy

Received August 6, 2010; Accepted October 13, 2010; Published December 7, 2010

Copyright: (c) 2010 Gold et al. This is an open-access article distributed under the terms of the Creative Commons Attribution License, which permits unrestricted use, distribution, and reproduction in any medium, provided the original author and source are credited.

Funding: This work was funded by SomaLogic. SomaLogic had no role in the original prospective CKD study. SomaLogic, with input from D.L. and T.P., designed the retrospective CKD study with archived samples, collected and analyzed the data, decided to publish, and prepared the manuscript. The National Institutes of Health (NIH) had no role in study design, data collection and analysis, decision to publish, or preparation of the manuscript.

Competing Interests: The authors have read the journal's policy and have the following conflicts: L Gold, D Ayers, J Bertino, C Bock, E Brody, J Carter, T Fitzwater, D Flather, A Forbes, T Foreman, C Fowler, B Gawande, M Goss, M Gunn, S Gupta, D Halladay, N Janjic, T Jarvis, S Jennings, E Katilius, T Keeney, N Kim, S Kraemer, N Le, B Lollo, W Mayfield, M Mehan, R Mehler, S Nelson, M Nikrad, U Ochsner, R Ostroff, M Otis, S Pietrasiewicz, D Resnicow, J Rohloff, G Sanders, D Schneider, B Singer, A Stewart, J Vaught, M Vrkljan, J Walker, M Watrobka, S Waugh, A Weiss, S Wilcox, S Wolk, C Zhang, and D Zichi are employed by SomaLogic. B Eaton and T Koch are consultants to SomaLogic. A Bock, A Dalby, B Eaton, J Heil, J Heilig, B Hicke, G Husar, L Kroiss, W Lindsey, M Nelson, D Nieuwlandt, S Sattin, M Stanton, A Sterkel, S Stratford, and A Wolfson are former employees of SomaLogic. SomaLogic has filed patent applications on aspects of this work. This does not alter the authors' adherence to all the PLoS ONE policies on sharing data and materials.

*E-mail: Igold@somalogic.com (LG); jwalker@somalogic.com (JJW) 


\section{Introduction}

Proteins present in blood are an immediate measure of an individual's phenotype and state of wellness. Secreted proteins, released from diseased cells and surrounding tissues, contain important biological information with the potential to transform early diagnostic, prognostic, therapeutic, and even preventative decisions in medicine.

We will realize the full power of proteomics only when we can measure and compare the proteomes of many individuals to identify biomarkers of human health and disease and track the blood-based proteome of an individual over time. Because the human proteome contains an estimated 20,000 proteins - plus splicing and post-translational variants - that span a concentration range of $\sim 12$ logs, identifying and quantifying valid biomarkers is a great technical challenge. Proteomic measurements demand extreme sensitivity, specificity, dynamic range, and accurate quantification.

The desire to profile the changes in protein expression at large scale is not new. Attempts at high-content proteomics began with 2-D gels and now mostly employ mass spectrometry (MS) and antibody-based technologies [1,2]. MS can deliver specific analytical capabilities and the technology has advanced remarkably in the past decade. Despite great promise for MS in clinical proteomics, many challenges remain including issues of sensitivity (typically $\mathrm{nM}$ in current approaches), specificity, reproducibility, throughput, and cost [2-9].

Antibody-based methods are more sensitive than 2-D gels or MS and can detect analytes in the sub-nM range due to the high affinity of antibodies for their targets (typically $\mathrm{nM}$ to $\mathrm{pM}$ ). However, non-specific binding of antibodies to non-cognate proteins, other macromolecules, and surfaces requires sandwichtype assays where the second antibody contributes to enhanced specificity through an independent binding event. In other words, technologies such as Enzyme-Linked Immuno-Sorbent Assays (ELISAs) attain high sensitivity by combining the specificity of two different antibodies to the same protein, requiring that both bind to elicit a signal [1]. Although broadly used in single-analyte tests, it has recently become clear that such assays cannot be multiplexed above a few tens of simultaneous measurements $[10,11]$ in large part because cross-reactivity of secondary antibodies to surface-immobilized proteins (including primary antibodies) dramatically erodes specificity [1]. This inherent characteristic compromises the performance of antibody-based arrays including printed antibodies, sandwich formats, and beadbased arrays $[1,12]$. A recently reported proximity ligation assay that relies on antibody sandwich formation in solution followed by ligation of antibody-tethered nucleic acids and PCR amplification has been multiplexed with six analytes [10].

To address these challenges, we set out to develop a proteomics array technology analogous to the highly successful nucleic acid hybridization microarray. To create this technology, we developed a new class of DNA-based aptamers enabled by a versatile chemistry technology that endows nucleotides with protein-like functional groups. These modifications greatly expand the repertoire of targets accessible to aptamers. The resulting technology provides efficient, large-scale selection of exquisite protein-binding reagents selected specifically for use in highlymultiplexed proteomics arrays. Aptamers are a class of nucleic acid-based molecules discovered twenty years ago [13,14] and have since been employed in diverse applications including therapeutics [15], catalysis [16], and now proteomics. Aptamers are short single-stranded oligonucleotides, which fold into diverse and intricate molecular structures that bind with high affinity and specificity to proteins, peptides, and small molecules [17-19]. Aptamers are selected in vitro from enormously large libraries of randomized sequences by the process of Systematic Evolution of Ligands by EXponential enrichment (SELEX) [13,14]. A SELEX library with 40 random sequence positions has $4^{40}\left(\sim 10^{24}\right)$ possible combinations and a typical selection screens $10^{14}-10^{15}$ unique molecules. This is on the order of $10^{5}$ times larger than standard peptide or protein combinatorial molecular libraries [20].

Based on the collective knowledge of the aptamer field that has developed since its inception [13,14], we hypothesized that aptamers could make exceptional reagents for high-content proteomics. There were many examples of high affinity RNA and DNA aptamers selected against human proteins [19]. However, there were also examples of difficult protein targets for which standard RNA and DNA SELEX did not yield high affinity aptamers. With two key innovations, we created a new class of aptamer, the Slow Off-rate Modified Aptamer (SOMAmer), which enabled efficient selection of high-affinity aptamers for almost any protein target and the development of a novel highly-multiplexed assay for high-performance proteomics. Here we present the development of these unique reagents in the context of our highcontent, high-performance, low-cost proteomics array, and demonstrate the potential of the platform to identify biomarkers from clinically-relevant samples.

\section{Results}

\section{SELEX with chemically-modified nucleotides}

The first innovation in developing the SOMAmer was motivated by the idea that aptamers can be endowed with protein-like properties by adding functional groups that mimic amino acid side-chains to expand their chemical diversity [21]. Eaton and colleagues developed the technology to efficiently synthesize nucleotides modified with diverse functional groups and to utilize them in SELEX [21,22]. This innovation was used to select catalysts, including the first RNA-catalyzed carbon-carbon bond formation [16,23]. Building on this work, we developed modified deoxyribonucleotides and SELEX methods [24] to select modified DNA aptamers from libraries that incorporate one of four dUTPs modified at the 5-position (Figure 1). This included

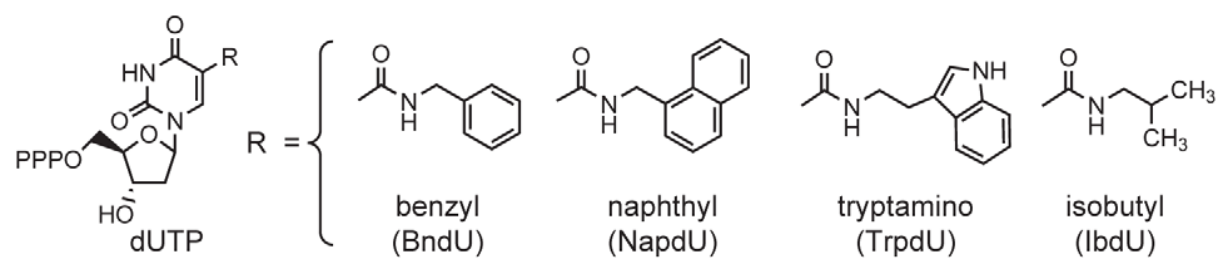

Figure 1. Modified Nucleotides. Nucleotide triphosphate analogs modified at the 5-position (R) of uridine (dUTP): 5-benzylaminocarbonyl-dU (BndU); 5-naphthylmethylaminocarbonyl-dU (NapdU): 5-tryptaminocarbonyl-dU (TrpdU); and 5-isobutylaminocarbonyl-dU (iBudU).

doi:10.1371/journal.pone.0015004.g001 
the synthesis of random libraries with modified nucleotides and the enzymatic amplification of SELEX pools that contain modified nucleotides (see Materials and Methods).

To test whether modified nucleotides improve SELEX, we compared selections with modified and unmodified nucleotides targeting thirteen "difficult" human proteins that had repeatedly failed SELEX with unmodified DNA. As a control, we included GA733-1 protein, which had yielded high-affinity aptamers with unmodified DNA SELEX. The results (Table 1) show that only SELEX with modified nucleotides yielded high-affinity aptamers to these difficult proteins. Different modifications worked better with different proteins. This shows that applying multiple modifications to the same target ensures a higher probability of success. Based on these results, we adopted modified nucleotide SELEX exclusively in our standard selections. To date, we have selected high-affinity aptamers (with most $\mathrm{K}_{\mathrm{d}}$ values lower than $\mathrm{nM}$, see Figure 2) to over 1000 human proteins, nearly all the proteins we have targeted. There are no obvious commonalities among those proteins that were initially unsuccessful in SELEX with unmodified DNA.

We first implemented 5-benzylaminocarbonyl-dU (BndU) in our high-throughput SELEX pipeline, and our success rate for selections to human proteins rose from $<30 \%$ to $>50 \%$ to a diversity of human proteins. This supported our hypothesis that we could develop one SELEX protocol that would work repeatedly for very different proteins. Since then, we have incorporated four modified nucleotides, BndU, 5-naphthylmethylaminocarbonyl-dU (NapdU), 5-tryptaminocarbonyl-dU (TrpdU), and 5-isobutylaminocarbonyl-dU (iBudU). Since the incorporation of these modified nucleotides into SELEX experiments, our overall success rate (pool $\mathrm{K}_{\mathrm{d}}<\sim 30 \mathrm{nM}$ ) is $\sim 84 \%(1204 / 1428)$ for high quality SOMAmers to a wide range of human proteins. The 813 human proteins measured by the current array are shown in Table S1. Overall, these results provide the first comprehensive evidence that

Table 1. SELEX library affinities $\left(\mathrm{K}_{\mathrm{d}},(\mathrm{M})\right)$ with unmodified and modified nucleotides.

\begin{tabular}{|c|c|c|c|c|}
\hline Target Protein & dT & benzyl-dU & isobutyl-dU & $\begin{array}{l}\text { tryptamino- } \\
\text { dU }\end{array}$ \\
\hline $4-1 B^{\S}$ & failed & $6 \times 10^{-9}$ & Failed & $4 \times 10^{-9}$ \\
\hline$B 7^{\S}$ & failed & $1 \times 10^{-8}$ & Failed & $7 \times 10^{-9}$ \\
\hline$B 7-2^{\S}$ & failed & Failed & Failed & $6 \times 10^{-9}$ \\
\hline CTLA-4 $4^{\S}$ & failed & Failed & Failed & $1 \times 10^{-9}$ \\
\hline sE-Selectin ${ }^{\S}$ & failed & Failed & Failed & $2 \times 10^{-9}$ \\
\hline $\begin{array}{l}\text { Fractalkine/ } \\
\text { CXC3L-1 }\end{array}$ & failed & Failed & Failed & $5 \times 10^{-11}$ \\
\hline GA733-1 protein $^{\S}$ & $9 \times 10^{-9}$ & $3 \times 10^{-9}$ & $5 \times 10^{-9}$ & $5 \times 10^{-10}$ \\
\hline gp130, soluble & failed & $6 \times 10^{-9}$ & $2 \times 10^{-8}$ & $1 \times 10^{-9}$ \\
\hline HMG-1 & failed & Failed & $2 \times 10^{-8}$ & $5 \times 10^{-9}$ \\
\hline IR & failed & $2 \times 10^{-9}$ & $1 \times 10^{-8}$ & $2 \times 10^{-10}$ \\
\hline Osteoprotegrin $\$$ & failed & $5 \times 10^{-9}$ & $9 \times 10^{-9}$ & $2 \times 10^{-10}$ \\
\hline PAI-1 & failed & $4 \times 10^{-10}$ & $9 \times 10^{-10}$ & $2 \times 10^{-10}$ \\
\hline P-Cadherin ${ }^{\S}$ & failed & $4 \times 10^{-9}$ & $5 \times 10^{-9}$ & $3 \times 10^{-9}$ \\
\hline sLeptin $\mathrm{R}^{\S}$ & failed & $2 \times 10^{-9}$ & Failed & $5 \times 10^{-10}$ \\
\hline
\end{tabular}

${ }^{5}$ The protein used was expressed as a fusion to the Fc of human IgG1. No detectable binding of the active library to an alternate Fc fusion proetin was observed.

doi:10.1371/journal.pone.0015004.t001

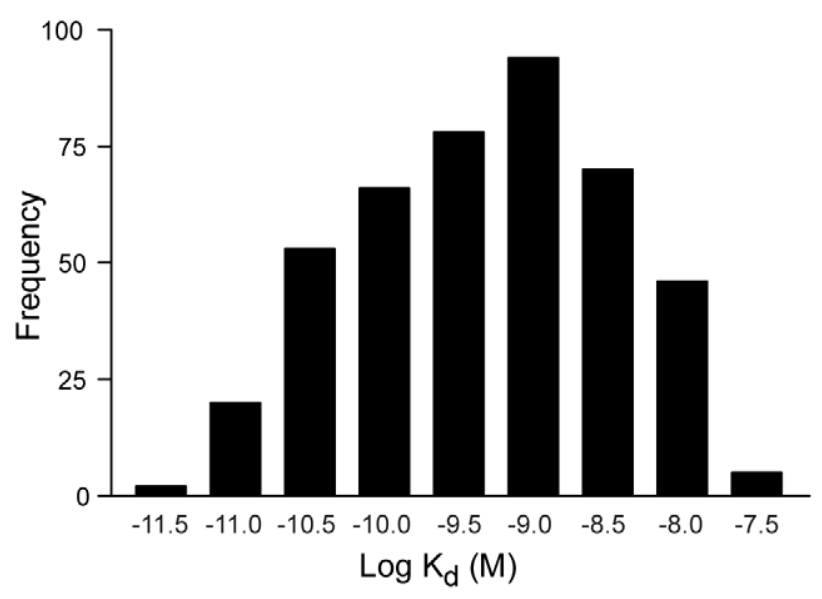

Figure 2. Dissociation constants. Distribution of dissociation constant $\left(\mathrm{K}_{\mathrm{d}}\right)$ values for 434 SOMAmers. doi:10.1371/journal.pone.0015004.g002

modified nucleotides can expand the range of possible aptamer targets and improve their binding properties.

\section{SELEX for slow off-rates}

The second innovation was a solution to the principal challenge of identifying a second element of specificity beyond binding of a second ligand for use in high-content arrays. Inspired by classic kinetic theory of specific binding in complex mixtures $[25,26]$, we employed kinetic manipulations to help overcome the problem of non-specific SOMAmer-protein binding. To achieve this second element of specificity, we selected for aptamers with slow dissociation rates $\left(t_{1 / 2}>30 \mathrm{~min}\right)$ that allow selective disruption of non-specific (or non-cognate) binding interactions by using a large excess of a polyanionic competitor.

For example, Figure 3 shows the half-life of dissociation of kallistatin, LBP, and TIG2 SOMAmers from their cognate targets (determined by using unlabeled SOMAmers) are 65, 44, and 65 minutes, compared to $<1$ minute for dissociation of the same SOMAmers from histone H1.2, a known DNA binding protein. Specific interactions are disrupted to a far lesser degree by dextran sulfate for all three SOMAmers. Figure 4 shows a representative distribution of dissociation half-lives for SOMAmers selected for our multiplexed proteomics assay.

\section{SOMAmer specificity}

The assay uses one SOMAmer per analyte rather than a sandwich of binding reagents and thus depends on equilibrium binding and kinetics for specificity. The difference in dissociation rates between cognate and non-cognate interactions contributes significantly to specificity in the assay (Figure 3). The use of sequential capture of protein-SOMAmer complexes on two sets of streptavidin beads, first through biotin-labeled SOMAmers (Catch-1) and then through biotin-labeled proteins (Catch-2), substantially reduces non-specific interactions. We assessed the specificity of select SOMAmers for the targets they were selected against in an affinity binding assay that mimics our multiplexed proteomics assay. The experimental method is outlined in Figure 5 and detailed in Materials and Methods. This experiment mimics Catch-1 and Catch-2 in the proteomics assay and then uses a third step to capture the bound SOMAmer-protein complex with an oligo that is complementary to a portion of the SOMAmer and acts as an affinity tag. This "Catch-3" step is analogous to the DNA microarray hybridization step in the proteomics assay. The 

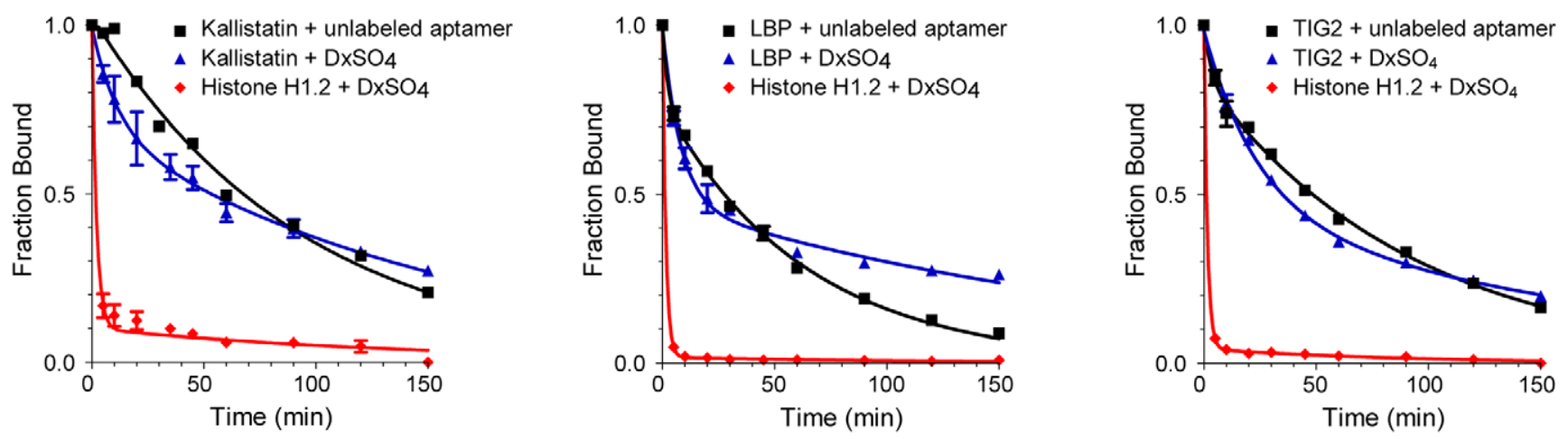

Figure 3. Kinetic discrimination between cognate and non-cognate interactions. Dissociation rate measurements for specific and nonspecific protein interactions with representative Kallistatin, LBP, and TIG2 SOMAmers. Histone H1.2 binds to random DNA sequences and was used to demonstrate non-specific binding. The fraction of radiolabeled SOMAmer (10 pM) bound to its cognate target is shown after addition of $50 \mathrm{nM}$ unlabeled SOMAmer (squares) or $0.3 \mathrm{mM}$ dextran sulfate (triangles) as a function of time. Rapid dissociation of non-specific complexes in the presence of $0.3 \mathrm{mM}$ dextran sulfate is also shown (diamonds).

doi:10.1371/journal.pone.0015004.g003

captured complexes are then disrupted and the proteins are eluted and analyzed by denaturing polyacrylamide gel electrophoresis (PAGE), as shown in Figure 6.

As shown in Figure 6, eluate from Catch-1 beads generally contains the target protein as well as several other proteins that bind SOMAmers non-specifically. Eluate from Catch-2 beads contains only the target protein in substantially pure form, along with its cognate SOMAmer (for these experiments, reversible protein attachment to monomeric avidin Catch-2 beads was used). This is likely due, in part, to a reduction in the amount of total protein following Catch-1 bead washing (only SOMAmer-bound or surface-bound proteins remain) as well as to release and recapture of complexes on separate beads in a reversed orientation (attachment through biotin on proteins).

To further assess the specificity of selected $(>20)$ SOMAmers for their target proteins, we excised the resulting PAGE gel samples (entire lanes) from the plasma affinity binding experiment described above and analyzed them by mass spectrometry (MS). In all cases, the results confirmed that the gel band contained the target protein (Table 2). This was evidenced by the identification of peptides that map to the target protein using their specific fragmentation patterns. Peptides that mapped to other proteins

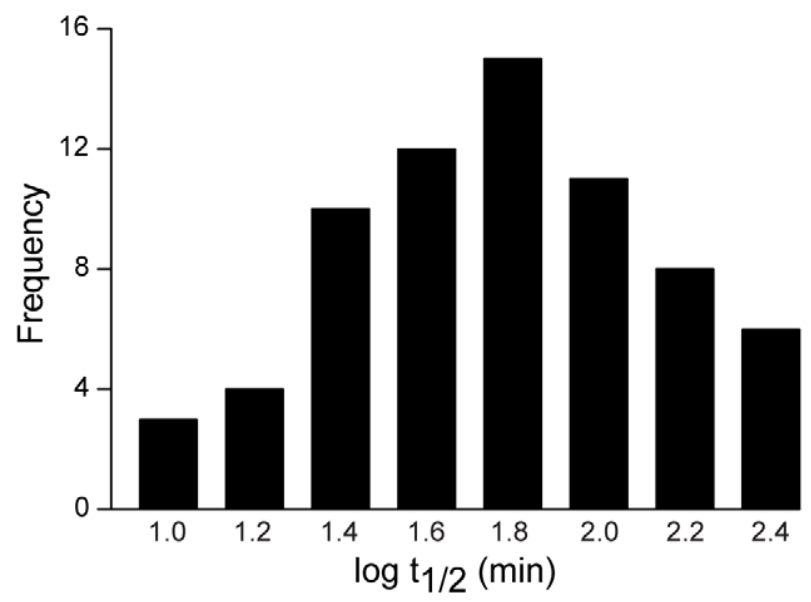

Figure 4. Dissociation rates. Distribution of dissociation rate $\left(t_{1 / 2}\right)$ values for 72 SOMAmers representative of those in proteomic arrays. doi:10.1371/journal.pone.0015004.g004 were also identified, although the number of spectra for these proteins was typically much lower than for the specific target. Such contaminants are a relatively small fraction because the PAGE gels show sharp uniform bands with very little background, which suggests that the majority of the material is the target protein.

\section{Multiplex proteomic assay}

To create a high-content proteomics platform for biomarker discovery, we developed a novel assay (Figure 7) which transforms a complex proteomic sample (e.g., plasma, serum, conditioned media, cell lysates, etc.) into a quantified protein signature. The assay leverages equilibrium binding and kinetic challenge [1]. Both are carried out in solution, not on a surface, to take advantage of more favorable kinetics of binding and dissociation [1]. The kinetic challenge works well for two reasons. First, dissociation rates of non-cognate SOMAmer-protein interactions are generally much faster (half-lives of a few minutes or less). Second, since all aptamers are polyanions, another polyanion at high concentration, such as dextran sulfate, can serve as a common competitor that dramatically minimizes rebinding events in a multiplex assay. In contrast, a common non-denaturing competitor of all antibodyantigen interactions or, more generally, protein-protein interactions, is not known.

An overview of the assay is shown in Figure 7. Briefly, the sample is incubated with a mixture of SOMAmers each containing a biotin, a photocleavable group, and a fluorescent tag followed by capture of all SOMAmer-protein complexes on streptavidin beads (Catch-1) (Figure 7A-C). After stringent washing of the beads to remove unbound proteins and labeling of bead-associated proteins with biotin under controlled conditions (Figure 7D), the complexes are released from the beads back into solution by UV light irradiation and diluted into a high concentration of dextran sulfate, an anionic competitor. The biotin that was originally part of the SOMAmer remains on beads. The anionic competitor coupled with dilution selectively disrupts non-cognate complexes (see Figure 7E) and because only the proteins now contain biotin, the complexes are re-captured on a second set of beads (Catch-2) from which unbound SOMAmers are removed by a second stringent washing (Figure 7F). The SOMAmers that remain attached to beads are eluted under high $\mathrm{pH}$-denaturing conditions and hybridized to sequence-specific complementary probes printed on a standard DNA microarray (Figure 7G,H). 
Catch 1

Streptavidin Agarose Beads
Catch 2

Monomeric Avidin beads
Catch 3

Primer Beads
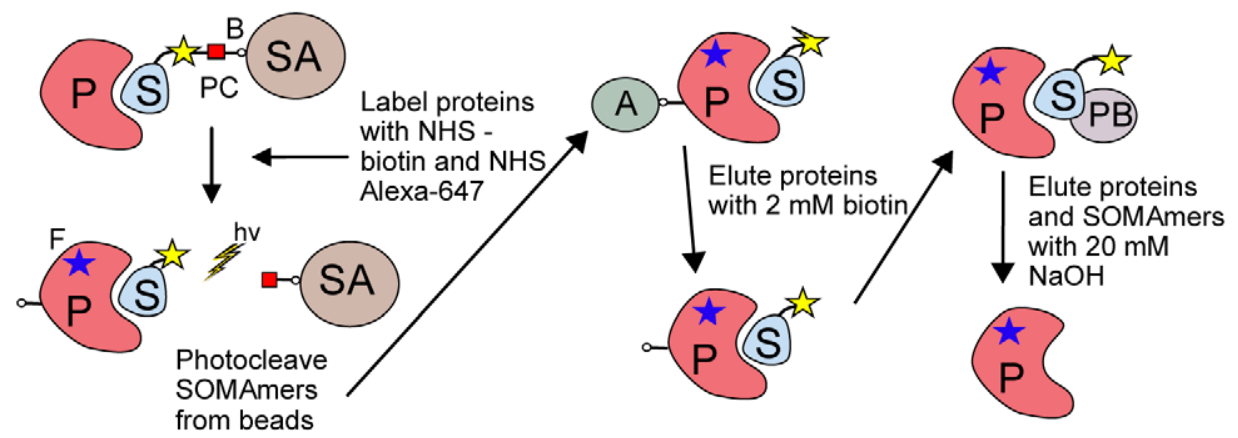

Figure 5. Affinity capture assay. SOMAmers are mixed with the target sample (purified protein or plasma) and incubated to bind to equilibrium. In Catch-1 bound SOMAmer(S)-protein(P) complexes are captured onto streptavidin beads (SA) and the proteins are tagged with biotin (B) (NHSbiotin) and fluorescent label (F) (NHS Alexa 647). Unbound proteins are washed away. Bound complexes are released from the beads by cleaving the photo-cleavable linker (PC) with ultraviolet light. In Catch-2 SOMAmer-protein complexes are captured onto monomeric avidin beads (A), washed, and eluted from the beads with $2 \mathrm{mM}$ biotin. At this stage, SOMAmer-protein complexes are subjected to a kinetic challenge analogous to that used in the proteomics assay. Specific complexes survive the challenge and non-specific complexes dissociate. In the final step, Catch-3, bound complexes are captured onto primer beads (PB) by DNA primer that is complementary to a portion of the SOMAmer and any remaining unbound protein resulting from the kinetic challenge is washed away. Finally, the captured complexes are dissociated with $20 \mathrm{mM} \mathrm{NaOH}$ and the target protein is eluted for analysis by PAGE.

doi:10.1371/journal.pone.0015004.g005

The result is a mixture of SOMAmers that quantitatively reflects protein concentrations in the original sample. The modified nucleotides in SOMAmers are designed to maintain canonical base-pairing [24,27] (in a DNA duplex, adducts at the 5position of pyrimidines are directed toward the major groove of DNA) and hybridize effectively to unmodified DNA oligonucleotides on the array (this is also required for replication during SELEX). The capture of SOMAmers on a hybridization array permits quantitative determination of the protein present in the original sample by converting the assay signal (relative fluorescence units, RFUs) to analyte concentration (Figure 8). Thus, our assay takes advantage of the dual nature of aptamers as molecules capable of both folding into complex three-dimensional structures, which is the basis of their unique binding properties, and hybridization to specific capture probes.

\section{Target protein menu}

The current version of our assay measures 813 human proteins (Table S1). These proteins represent a wide range of sizes, physicochemical properties, such as a pI range of 4-11 (Figure 9), and biological functions from a variety of molecular pathways and gene families (Figure 10). Thus, SOMAmer technology enables an efficient and scalable pipeline to generate unbiased content for proteomics arrays.

\section{Assay performance}

Reproducibility. To assess the reproducibility of the assay, we collected serum and plasma samples from 18 healthy volunteers and assayed five replicates of each sample in a single run, and repeated this in triplicate. The results show an overall low median $\mathrm{CV}$ of $\sim 5 \%$ for intra-run and inter-run $\mathrm{CV}$. The $\mathrm{CV}$ for each SOMAmer was computed for each sample by averaging over the replicates and then averaging these $\mathrm{CVs}$ over all the samples. Both intra- and inter-plate $\mathrm{CVs}$ were computed for each dilution mix and are shown in Figure S1.

Limits of quantification. To assess the quantitative performance of the assay, we measured the upper and lower limit of quantification (ULOQ and LLOQ) and dynamic range of quantification (ROQ) values for a representative subset (356) target proteins. The LOQ experiments measured six-point standard curves spanning six logs in concentration, from $10 \mathrm{nM}$ to $10 \mathrm{fM}$, in buffer. Overall, the median LLOQ was $\sim 1 \mathrm{pM}$, with some as low as $100 \mathrm{fM}$, the median ULOQ was $\sim 1.5 \mathrm{nM}$, and the median ROQ was $\sim 3$ logs (results summarized in Figure S2 and complete results reported in Table S2). We conducted these experiments in buffer because many target proteins have endogenous blood concentrations that prevent determining precision profiles and LOQs. For some proteins with low endogenous concentrations, we found consistent performance when titrated into $10 \%$ plasma (see below).

For each analyte, we generated a precision profile, which shows the variation in $\% \mathrm{CV}$ for calculated concentration as a function of analyte concentration. Precision profiles provide an analytic measurement of assay performance and establish ULOQ LLOQ and ROQ. In some cases the measurements did not plateau in the upper measured range $(10 \mathrm{nM})$ and the reported values represent a minimum estimate of ULOQ and resulting ROQ. A typical doseresponse curve and precision profile from the data set is shown in Figure S3. We computed a full precision profile for each analyte using two methods. One method modeled the standard deviation for calculated concentrations $\sigma_{x}$ directly, and the other method modeled $\sigma_{\log R F U}$ from which $\sigma_{x}$ was computed. For the example shown in Figure S3, both methods gave similar results. This particular analyte shows a remarkable five-log quantification range at a 20\% CV cutoff with an LLOQ of $0.4-0.6 \mathrm{pM}$ and a ULOQ of $40-50 \mathrm{nM}$. In general there is good agreement between the two different methods for computing precision profiles, and the assay response $\sigma_{\log R F U}$ method was used to calculate the values shown in Table S2.

Buffer versus plasma LOQs. To compare assay performance for measuring analytes in buffer to measuring proteins in plasma, we measured LLOQs, ULOQs, and ROQs in buffer and plasma for twenty-eight analytes with low endogenous plasma concentrations. The results are summarized in Figure S4 and detailed in Table S3. Comparing these measurements, the average log ratio of plasma to buffer LLOQs and ULOQs was 0.26 and 0.32 , which shows that the average LLOQ and ULOQ for these proteins was 2-3 fold higher in plasma than buffer. Of the 28 proteins compared, about two thirds 


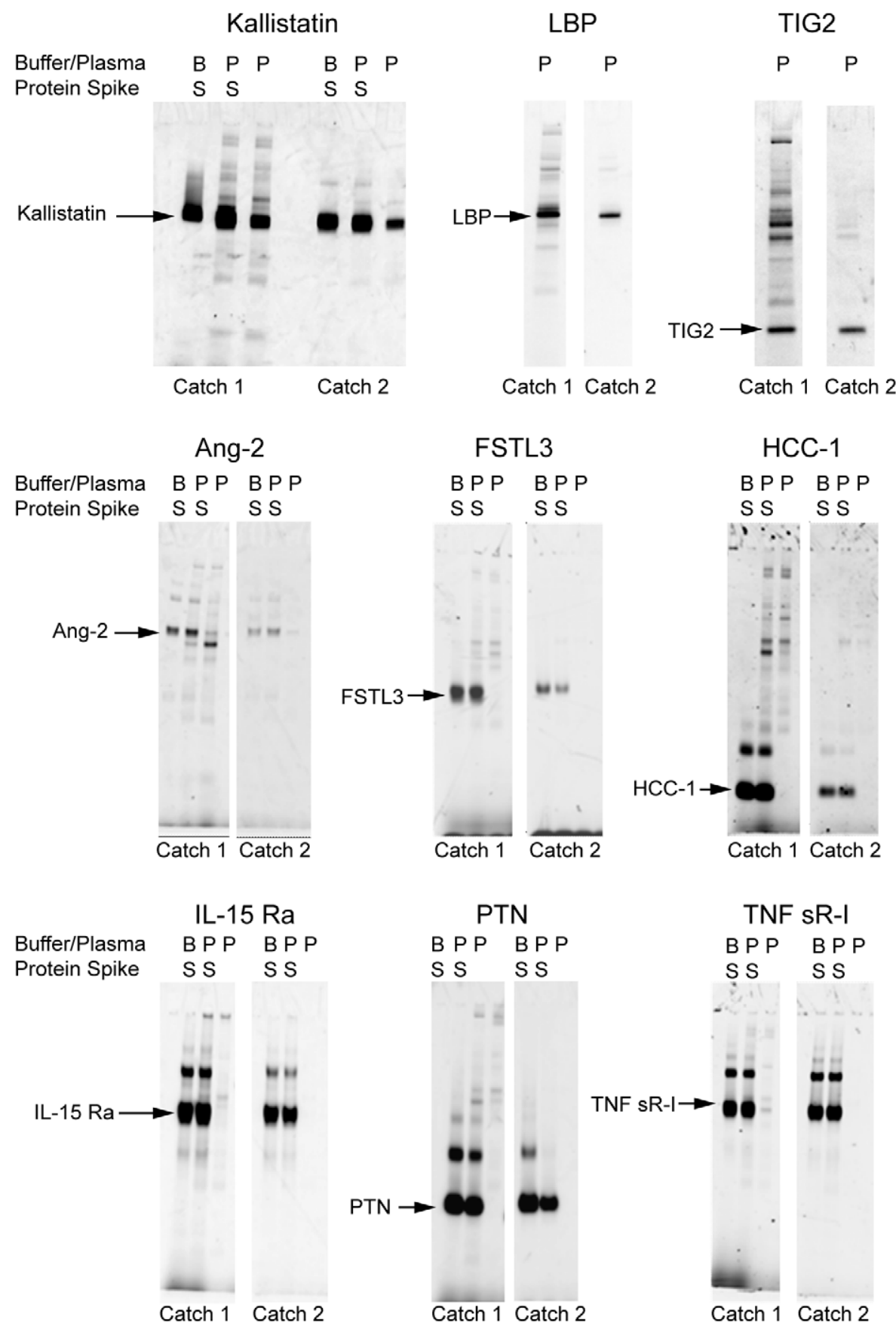

Figure 6. Affiinity capture of representative SOMAmer protein targets. SDS-PAGE visualization of representative SOMAmer protein targets p Kallistatin, LBP and TIG2. The Kallistatin gel shows proteins bound to the Kallistatin SOMAmer for target added to buffer (lane 1), target added to 10\% plasma (lane 2), and 10\% plasma alone (lane 3). The first set of three lanes demonstrates all of the proteins eluted from Catch-1 beads. The second set of lanes shows the SOMAmer-bound proteins eluted from Catch-2 beads. The LBP and TIG2 gels demonstrate proteins recovered from $10 \%$ plasma using the LBP and TIG2 SOMAmers, respectively (without adding proteins to plasma for these three gels). The endogenous plasma proteins captured by the Kallistatin, LBP, and TIG2 SOMAmers were identified by LC-MS/MS as the intended target proteins (Table 2). The remaining gels show affinity capture assays for CKD-related proteins. For each example the gel shows the results for purified target protein spiked into buffer (lane 1), purified target protein spiked into 10\% plasma (lane 2), and 10\% plasma (lane 3). The first set of three lanes demonstrates all of the proteins eluted from Catch-1 beads. The second set of lanes shows the aptamer-bound proteins eluted from Catch-2 beads. doi:10.1371/journal.pone.0015004.g006

(20/28) had somewhat higher LLOQs in plasma and about one third $(8 / 28)$ had lower LLOQs in plasma compared to buffer. Overall, these results suggest that buffer precision profiles provide a reasonable assessment of the quantitative behavior of the multiplexed assay in plasma.

\section{Example: Chronic kidney disease}

To demonstrate the utility of the platform to discover diseaserelated biomarkers, we analyzed plasma from subjects with chronic kidney disease (CKD), the slow loss of kidney function over time. CKD is a recently recognized global public health 
Table 2. Identification by LC-MS/MS of affinity captured proteins.

\begin{tabular}{lllllll}
\hline Protein & Accession & Protein ID Probability & Unique Peptides & Unique Spectra & Total Spectra & \%Sequence Coverage \\
\hline Kallistatin & IPI00328609 & 1 & 22 & 31 & 39 & 53.6 \\
LBP & IPI00032311 & 1 & 13 & 16 & 22 & 27.0 \\
TIG2 & IPI00019176 & 0.95 & 2 & 2 & 2 & 13.5 \\
\hline
\end{tabular}

doi:10.1371/journal.pone.0015004.t002

problem that is "common, harmful, and treatable" with an estimated prevalence of nearly $10 \%$ worldwide [28]. Early intervention in CKD can substantially improve prognosis, which is otherwise poor [28-31]. To achieve early diagnosis, predictive, non-invasive CKD biomarkers are needed. CKD biomarkers could also be useful for monitoring disease progression and guiding treatment [28-31].

We chose CKD as a test case because kidney physiology provides filtration of serum molecules based on size (molecular mass) and charge [32] - thus CKD might lead to an increase in the concentration of small proteins $(\mathrm{MW}<45 \mathrm{kDa})$. Disease progression is expected to be accompanied by an overall increase in plasma concentration of small proteins.

We obtained and analyzed plasma samples from 42 subjects with CKD. Eleven subjects had early-stage CKD based on estimated GFR (Table 3) (eGFR, defined as stages 1-2, median creatinine clearance $70 \mathrm{ml} / \mathrm{min} / \mathrm{m}^{2}$, range $62-97 \mathrm{ml} / \mathrm{min} / \mathrm{m}^{2}$ ) and 31 had late-stage CKD (stages 3-5, median creatinine clearance $25 \mathrm{ml} / \mathrm{min} / \mathrm{m}^{2}$, range $7-49 \mathrm{ml} / \mathrm{min} / \mathrm{m}^{2}$ ) [33]. We measured 614 human proteins (array size at the time analyses were conducted) simultaneously for each sample and compared the results of early- to late-stage CKD (Figure 11).

We identified 60 proteins that varied significantly between the two groups, using the Mann-Whitney test, with a q-value (false discovery rate-corrected p-value) of $4.2 \times 10^{-4}$ (Table S4). Eleven proteins with the most highly significant variation (q-values $<3.5 \times 10^{-7}$ ) are highlighted in Figure 12 and shown in Table 4 . Nine out of eleven are relatively small proteins $(<25 \mathrm{kDa})$. For all eleven proteins, there is an inverse correlation between eGFR (a marker of CKD progression) and protein concentration, suggesting they could be potential biomarkers of CKD progression (Figure 12). Two of the eleven proteins, cystatin $\mathrm{C}$ and $\beta_{2}$-microglobulin, are important known biomarkers of CKD [29-31] and two additional proteins, complement factor D and TNF sR-I, have been reported to have elevated concentrations in CKD $[34,35]$.

Accumulation in plasma of some small proteins appears to be a major change in the proteome. However, the concentration of many low molecular weight proteins did not change appreciably with disease progression (Figure 13); pI also was uncorrelated with an increase in plasma concentration as a function eGFR (data not shown). The surprising fact that the biomarkers are not simply ranked according to their molecular masses shows that reduced kidney function is complex. The accumulation of some (but not all) low molecular weight proteins, sometimes called "middle molecules", in plasma of patients with impaired renal filtration has long been implicated in the pathology of kidney disease[36]. Highcontent proteomic analysis provides a means of unbiased discovery of such proteins and their relationship to disease progression.

\section{Discussion}

We developed a new aptamer-based proteomic technology capable of measuring thousands of proteins in small volumes of biological samples with low limits of detection, a broad dynamic range, and high reproducibility. The current assay measures 813 proteins with $1 \mathrm{pM}$ median LLOQ, 7-log overall dynamic range $(\sim 100 \mathrm{fM}-1 \mu \mathrm{M})$ with three sample dilutions that span $\sim 2.5$ logs, and $5 \%$ median $\mathrm{CV}$. The content of the discovery assay is flexible and highly scalable, permitting the addition of content as the target menu grows.

To achieve this performance, we developed a new class of DNA-based aptamer, the Slow Off-rate Modified Aptamer (SOMAmer), based on two key innovations: novel chemicallymodified nucleotides that mimic amino acid side chains and new SELEX strategies to select aptamers with very slow off-rates. With this technology, our success rate for selecting high-quality aptamers to target proteins rose from $<30 \%$ to $>90 \%$ today. To date, we have selected high-quality SOMAmers to $>1,000$ human proteins.

We demonstrated the utility of our proteomics platform in biomarker discovery with a study of GKD. We identified 60 proteins that varied significantly between early and late stage GKD, which could provide a foundation for developing CKD diagnostics. In a study of more than 500 additional patients at risk for cardiovascular disease (whose eGFRs were also determined), we confirmed and extended the biomarkers associated with reduced filtration in this first CKD study (data not shown). These results provide further evidence for the validity of these potential CKD biomarkers, and further validation studies are in progress.

Overall, these results show that our multiplexed proteomics assay has the requisite reproducibility, sensitivity, and range for high-content proteomics studies and unbiased biomarker discovery.

We recognize that there are some limitations to the work presented here, which only demonstrates the specificity of SOMAmers for the proteins they were selected against. In order to further validate and standardize SOMAmer-based measurements, we plan studies with reference standards and other analytical methods, such as the affinity capture-MS method presented here. We are also expanding these studies to understand the specificity of SOMAmers for close homologues and alternate forms, such as the products of alternative splicing, posttranslational modifications, and proteolytic cleavage. We believe that SOMAmers will be highly specific, given our previous experience with highly-specific aptamers including, for example, the drug Pegaptanib (Macugen) for the treatment age-related macular degeneration, which binds specifically to VEGF121 but not VEGF165 [37,38], and an aptamer that distinguishes theophylline from caffeine, molecules that differ by just one methyl group [39].

In addition to the work described here, we have conducted clinical studies with our technology and discovered potential biomarkers in many areas with unmet medical need including cancer, cardiovascular conditions, neurological disorders, and infectious diseases. Frequently, the distributions of biomarker concentrations among two populations overlap to some degree, 

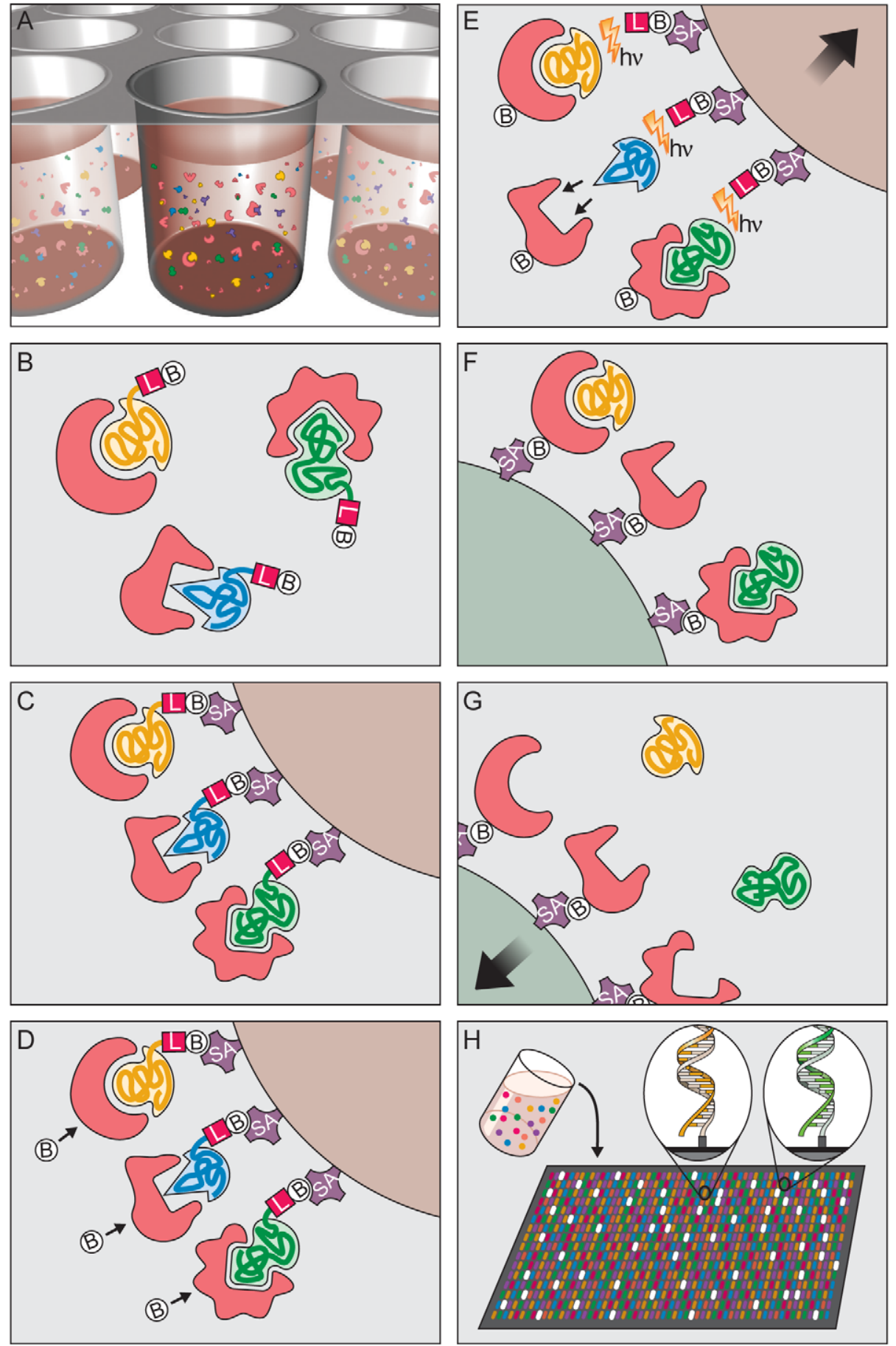

Figure 7. Principle of multiplex SOMAmer affinity assay. (A) Binding. SOMAmers and samples are mixed in 96-well microwell plates and allowed to bind. Cognate and non-cognate SOMAmer-target protein complexes form. Free SOMAmer and protein are also present. (B-H) Schematic sequence of assay steps leading to quantitative readout of target proteins. (B) SOMAmer-protein binding: DNA-based SOMAmer molecules (gold, blue, and green) have unique shapes selected to bind to a specific protein. SOMAmers contain biotin (B), a photo-cleavable linker (L) and a fluorescent tag at the $5^{\prime}$ end. Most SOMAmers (gold and green) bind to cognate proteins (red), but some (blue) form non-cognate complexes. (C) Catch-1. SOMAmers are captured onto a bead coated with streptavidin (SA) which binds biotin. Un-complexed proteins are washed away. (D) Proteins are tagged with NHS-biotin. (E) Photocleavage and kinetic challenge. UV light (hv) cleaves the linker and SOMAmers are released from beads, leaving biotin on bead. Samples are challenged with anionic competitor (dextran sulfate). Non-cognate complexes (blue SOMAmer) preferentially dissociate. (F) Catch-2 SOMAmer-protein complexes are captured onto new avidin coated beads by protein biotin tag. Free SOMAmers are washed away. (G) SOMAmers are released from complexes into solution at high $\mathrm{pH}$. (H) Remaining SOMAmers are quantified by hybridization to microarray containing single-stranded DNA probes complementary to SOMAmer DNA sequence, which form a double-stranded helix. Hybridized SOMAmers are detected by fluorescent tags when the array is scanned. doi:10.1371/journal.pone.0015004.g007 

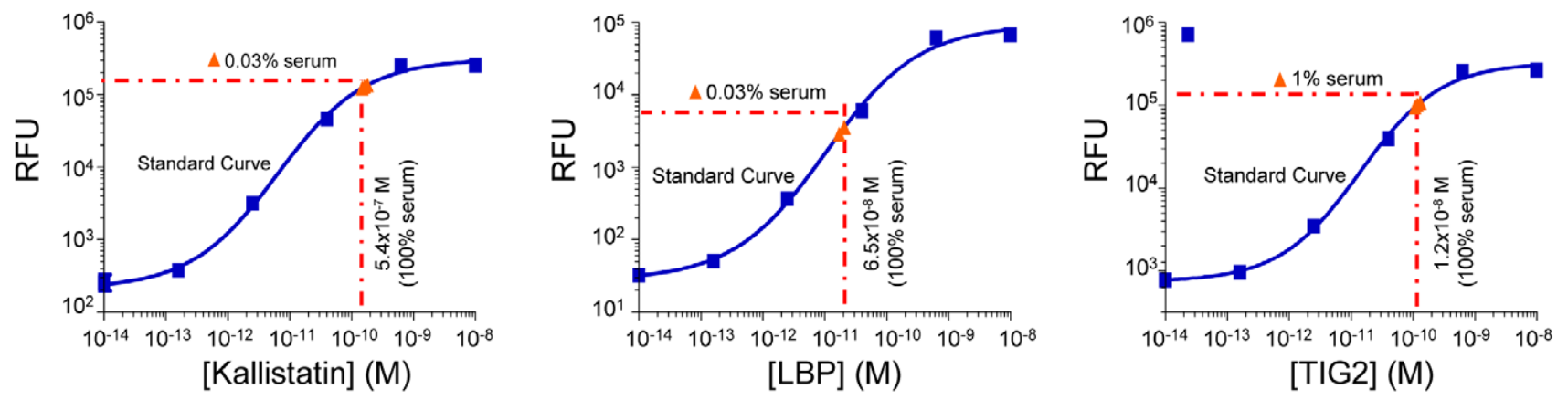

Figure 8. Proteomic assay standard curves. Each plot shows the standard curve for eight replicates of target spiked into buffer (blue squares). Triplicate measurements from diluted normal serum (red triangles, measured dilution indicated) are plotted onto the standard curve, and the calculated normal concentrations in $100 \%$ serum are shown.

doi:10.1371/journal.pone.0015004.g008

which creates the impetus to combine multiple biomarkers to achieve the most accurate diagnosis. In a related paper, we report the first large-scale application of this technology to discover and verify novel biomarkers for lung cancer in one of the most comprehensive proteomic biomarker studies published to date $[40]$.

\section{Materials and Methods}

\section{Ethics Statement}

All studies of human subjects were conducted with written informed consent. Both the original study of CKD and the biomarker study reported here were approved by the Institutional Review Board at Weil Medical College of Cornell University.

\section{SOMAmer development and SELEX}

Selection methods have been developed for use with poly-Histagged, biotinylated, and non-tagged proteins. Many variations on these protocol have been used to select the $>800$ SOMAmers for the proteomic platform, such as alternating selection conditions to increase stringency for slow off-rate SOMAmers or performing the equilibrium steps in solution rather than with targets pre- immobilized. The following protocol is representative and was used for the selection described for the results shown in Table 1. Selection methods are further detailed in our patents and published patent applications [41,42].

Preparation Modified Nucleotides. Modified nucleotides were manufactured by SomaLogic, Inc. with methods described by Vaught et al. [24].

Preparation of Candidate Mixtures. Candidate mixtures were prepared with dATP, dGTP, 5-methyl-dCTP (MedCTP) and either dTTP or one of three dUTP analogs: 5benzylaminocarbonyl-dU (BndU), 5-tryptaminocarbonyl-dU = TrpdU, and 5-isobutylaminocarbonyl-dU (iBudU) (Figure 1). Candidate mixtures were prepared by polymerase extension of a primer annealed to a biotinylated template. Several enzymes were screened for the ability to incorporate these modified nucleotides, as well as to amplify a modified template. We used Thermococcus kodakaraensis (KOD) DNA polymerase for PCR with a slightly modified buffer, although at low efficiency. Additionally, conditions have been determined to amplify selected DNA using a two-step process to avoid potential amplification biases. For each candidate mixture composition, $4.8 \mathrm{nmol}$ forward PCR primer and $4 \mathrm{nmol}$ template were combined in $100 \mu \mathrm{L} 1 \mathrm{X}$ KOD XL

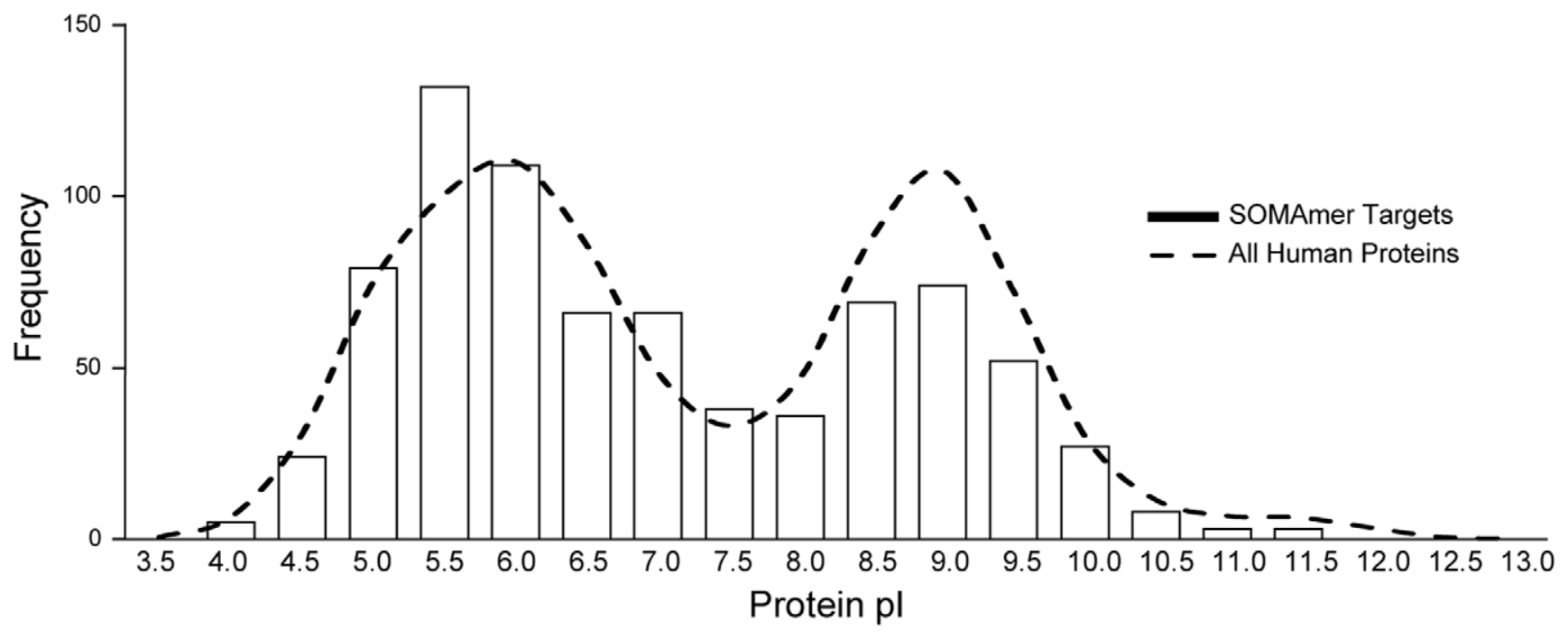

Figure 9. Target isoelectric points. Distribution of isoelectric points (pl) of proteins for which SOMAmers have been selected (bars) and of all human protein chains in UniProt (dashed line).

doi:10.1371/journal.pone.0015004.g009 


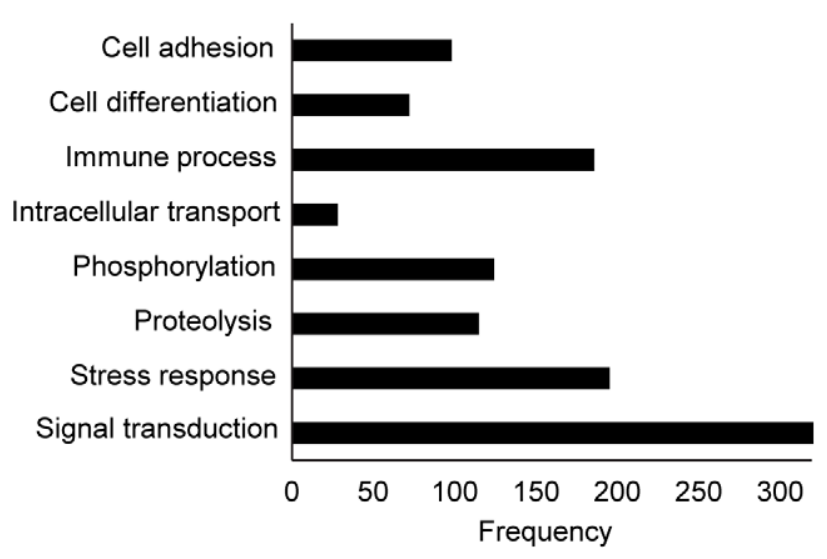

Figure 10. Protein target menu gene ontology. Distribution of most common gene ontology terms associated with the proteins measured by the current array.

doi:10.1371/journal.pone.0015004.g010

DNA Polymerase Buffer (EMD Chemicals), heated to $95^{\circ} \mathrm{C}$ for 8 minutes, and cooled on ice. Each $100 \mu \mathrm{L}$ primer:template mixture was added to a $400 \mu \mathrm{L}$ extension reaction containing $1 \mathrm{X}$ KOD DNA Polymerase Buffer, 0.125 U/ $\mu \mathrm{L}$ KOD DNA Polymerase, and $0.5 \mathrm{mM}$ each dATP, MedCTP, dGTP, and dTTP or dUTP analog, and incubated at $70^{\circ} \mathrm{C}$ for 30 minutes. Double-stranded product was captured via the template strand biotins by adding $1 \mathrm{~mL}$ streptavidin-coated magnetic beads (MagnaBind Streptavidin, Pierce, $5 \mathrm{mg} / \mathrm{mL}$ in $1 \mathrm{M} \mathrm{NaCl}+0.05 \%$ TWEEN20) and incubating at $25^{\circ} \mathrm{C}$ for 10 minutes with mixing. Beads were washed three times with $0.75 \mathrm{~mL}$ SBlT Buffer $(40 \mathrm{mM}$ HEPES, pH 7.5, $125 \mathrm{mM} \mathrm{NaCl}, 5 \mathrm{mM} \mathrm{KCl}, 1 \mathrm{mM} \mathrm{MgCl}_{2}$, $1 \mathrm{mM} \mathrm{CaCl}_{2}, 0.05 \%$ TWEEN-20). The SOMAmer strand was eluted from the beads with $1.2 \mathrm{~mL} 20 \mathrm{mM} \mathrm{NaOH}$, neutralized with $0.3 \mathrm{~mL} 80 \mathrm{mM} \mathrm{HCl}$, and buffered with $15 \mu \mathrm{L} 1$ M HEPES, $\mathrm{pH}$ 7.5. Candidate mixtures were concentrated with a Centricon30 to approximately $0.2 \mathrm{~mL}$, and quantified by UV absorbance spectroscopy.

Immobilization of Target Proteins. Target proteins were purchased with $(\mathrm{His})_{6}$ tags from the following vendors: AnaSpec, APE-Bridgepath ARP, Athens Research and Technology, BBridge International, Inc, Biogenesis, Calzyme, EMD Biosciences, Enzyme Research Laboratories, Invitrogen, Millipore, Nexomics, Pepro Tech, Peptide Institute, Inc., ProSci, ProSpec, ProteinX Lab, Proteome Resources, LLC, Quality Biological, Quidel, R\&D Systems, Research Diagnostics, RZPD GmbH, Sigma-Aldrich,

Table 3. Population demographics for chronic kidney disease study.

\begin{tabular}{lll}
\hline & & \\
\hline & Early stage CKD & Late stage CKD \\
\hline $\mathrm{N}$ (total =42) & 11 & 31 \\
Gender \%F (F/M) & $33 \%(4 / 11)$ & $45 \%(14 / 31)$ \\
Age (avg. yrs) & $62[51-68]$ & $67[57-77]$ \\
$\begin{array}{l}\text { Wt. (avg. kg) } \\
\text { BMl (avg.) }\end{array}$ & $89[73-98]$ & $88[75-104]$ \\
eGFR (median) & $30.5[26.6-36.5]$ & $31.8[27.1-36.6]$ \\
\hline $\begin{array}{l}\text { §Estimated glomerular filtration from creatinine clearance (MDRD formula) ml/ } \\
\text { min/m². } \\
\text { doi:10.1371/journal.pone.0015004.t003 }\end{array}$ & $70[62-97]$ & $25[7-49]$ \\
\hline
\end{tabular}

United States Biological, Upstate Biotechnology, and VWR. Proteins were immobilized on $\mathrm{Co}^{+2}$-NTA paramagnetic beads (MyOne TALON beads, Invitrogen). Target proteins were diluted to $0.2 \mathrm{mg} / \mathrm{mL}$ in $0.5 \mathrm{~mL} \mathrm{~B} / \mathrm{W}$ Buffer $(50 \mathrm{mM} \mathrm{Na-phosphate,}$ $\mathrm{pH} 8.0,300 \mathrm{mM} \mathrm{NaCl}, 0.01 \%$ TWEEN-20), and added to $0.5 \mathrm{~mL}$ MyOne TALON beads (pre-washed three times with B/ $\mathrm{W}$ Buffer and resuspended to $10 \mathrm{mg} / \mathrm{mL}$ in $\mathrm{B} / \mathrm{W}$ Buffer). The mixture was rotated for 30 minutes at $25^{\circ} \mathrm{C}$ and stored at $4^{\circ} \mathrm{C}$ until use. MyOne TALON beads coated with $(\mathrm{His})_{6}$ peptide were also prepared and stored as above. Prior to use, beads were washed 3 times with $\mathrm{B} / \mathrm{W}$ Buffer, once with $\mathrm{SB} 1 \mathrm{~T}$, and resuspended in SB1T.

SOMAmer Selection. Affinity selections were performed separately with each candidate mixture, comparing binding between target protein beads (signal) and $(\mathrm{His})_{6}$ beads (background). For each sample, a $0.5 \mu \mathrm{M}$ candidate DNA mixture was prepared in $40 \mu \mathrm{L}$ SB1T. $1 \mu \mathrm{L}$ of $1 \mathrm{mM}$ competitor oligo was added to the DNA, along with $10 \mu \mathrm{L}$ of a protein competitor mixture $(0.1 \% \mathrm{HSA}, 10 \mu \mathrm{M}$ casein, and $10 \mu \mathrm{M}$ prothrombin in SB1T).

Binding reactions were performed by adding $50 \mu \mathrm{L}$ target protein-coated beads or $(\mathrm{His})_{6}$-coated beads $(5 \mathrm{mg} / \mathrm{mL}$ in $\mathrm{SB} 1 \mathrm{~T})$ to the DNA mixture and incubating at $37^{\circ} \mathrm{C}$ for 15 minutes with mixing. The DNA solution was removed and the beads were washed 5 times at $37^{\circ} \mathrm{C}$ with SB1T containing $0.1 \mathrm{mg} / \mathrm{mL}$ herring sperm DNA (Sigma Aldrich). Unless indicated, all washes were performed by resuspending the beads in $100 \mu \mathrm{L}$ wash solution, mixing for 30 seconds, separating the beads with a magnet, and removing the wash solution. Bound SOMAmers were eluted from the beads by adding $100 \mu \mathrm{L}$ SB1T $+2 \mathrm{M}$ Guanidine-HCl and incubating at $37^{\circ} \mathrm{C}, 5$ minutes with mixing. The SOMAmer eluate was transferred to a new tube after magnetic separation. After the first two selection rounds, the final two of five target beads washes were done for 5 minutes instead of 30 seconds.

Primer beads were prepared by immobilizing biotinylated reverse PCR primer to streptavidin-coated paramagnetic beads (MyOne Streptavidin, Invitrogen). $5 \mathrm{~mL}$ MyOne Streptavidin beads $(10 \mathrm{mg} / \mathrm{mL})$ were washed once with $\mathrm{NaClT}$ ( $5 \mathrm{M} \mathrm{NaCl}$, $0.01 \%$ TWEEN-20), and resuspended in $5 \mathrm{~mL}$ biotinylated reverse PCR primer $(5 \mu \mathrm{M}$ in NaCIT). The sample was incubated at $25^{\circ} \mathrm{C}$ for 15 minutes, washed twice with $5 \mathrm{~mL}$ NaClT, resuspended in $12.5 \mathrm{~mL} \mathrm{NaClT}(4 \mathrm{mg} / \mathrm{mL})$, and stored at $4^{\circ} \mathrm{C}$.

Twenty-five $\mu \mathrm{L}$ of primer beads $(4 \mathrm{mg} / \mathrm{mL}$ in NaClT) were added to the $100 \mu \mathrm{L}$ SOMAmer solution in Guanidine Buffer and incubated $50^{\circ} \mathrm{C}, 15$ minutes with mixing. The SOMAmer solution was removed, and the beads were washed 5 times with SB1T. SOMAmer was eluted from the beads by adding $85 \mu \mathrm{L} 20 \mathrm{mM}$ $\mathrm{NaOH}$ and incubating at $37^{\circ} \mathrm{C}$ for 1 minute with mixing. $80 \mu \mathrm{L}$ SOMAmer eluate was transferred to a new tube after magnetic separation, neutralized with $20 \mu \mathrm{L} 80 \mathrm{mM} \mathrm{HCl}$, and buffered with $1 \mu \mathrm{L} 0.5 \mathrm{M}$ Tris-HCl, $\mathrm{pH} 7.5$.

SOMAmer Amplification and Purification. Selected SOMAmer DNA was amplified and quantified by QPCR. $48 \mu \mathrm{L}$ DNA was added to $12 \mu \mathrm{L}$ QPCR Mix (5X KOD DNA Polymerase Buffer, $25 \mathrm{mM} \mathrm{MgCl}_{2}, 10 \mu \mathrm{M}$ forward PCR primer, $10 \mu \mathrm{M}$ biotinylated reverse PCR primer, 5X SYBR Green I, $0.125 \mathrm{U} / \mu \mathrm{L}$ KOD DNA Polymerase, and $1 \mathrm{mM}$ each dATP, dCTP, dGTP, and dTTP) and thermal cycled in an ABI 5700 QPGR instrument (Applied Biosystems) with the following protocol: 1 cycle of $99.9^{\circ} \mathrm{C}, 15$ seconds, $55^{\circ} \mathrm{C}, 10$ seconds, $70^{\circ} \mathrm{C}$, 30 minutes; 30 cycles of $99.9^{\circ} \mathrm{C}, 15$ seconds, $72^{\circ} \mathrm{C}, 1$ minute. Quantification was done with the instrument software and the number of copies of DNA selected with target beads and (His) 6 beads were compared to determine signal/background ratios. 




Figure 11. Biomarker discovery in CKD. Distribution of the false discovery rate (q-value) for the Mann-Whitney test statistic comparing late-stage vs. early-stage CKD for each protein measured (indicated as a bar on the $\mathrm{x}$-axis) ordered arbitrarily.

doi:10.1371/journal.pone.0015004.g011

Following amplification, the PCR product was captured on MyOne Streptavidin beads via the biotinylated antisense strand. $1.25 \mathrm{~mL}$ MyOne Streptavidin beads $(10 \mathrm{mg} / \mathrm{mL})$ were washed twice with $0.5 \mathrm{~mL} 20 \mathrm{mM} \mathrm{NaOH}$, once with $0.5 \mathrm{~mL} \mathrm{SB} 1 \mathrm{~T}$, resuspended in $2.5 \mathrm{~mL} 3 \mathrm{M} \mathrm{NaCl}$, and stored at $4^{\circ} \mathrm{C}$. $25 \mu \mathrm{L}$ MyOne Streptavidin beads $(4 \mathrm{mg} / \mathrm{mL}$ in $3 \mathrm{M} \mathrm{NaCl})$ were added
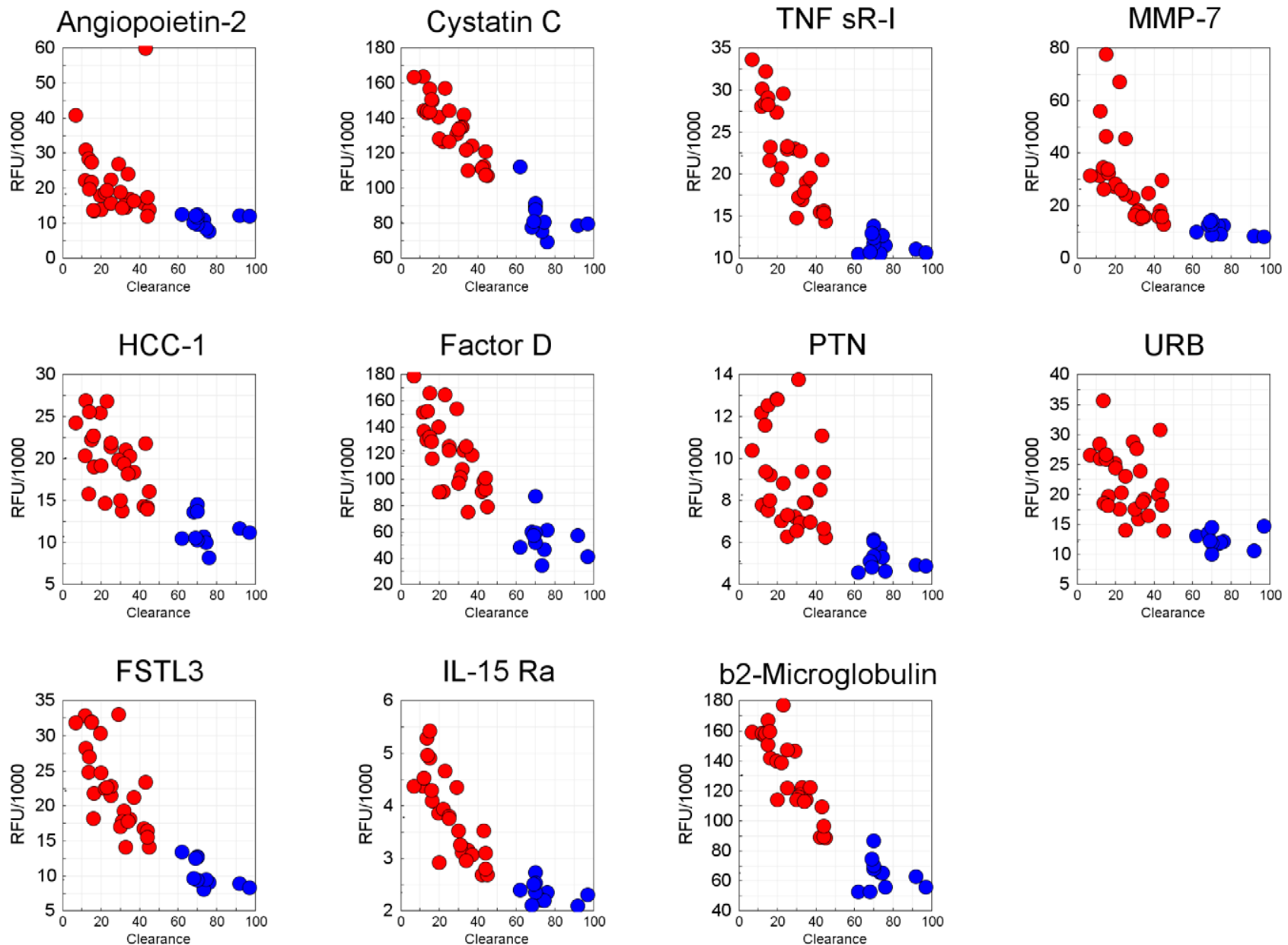

Figure 12. Potential CKD biomarkers. Eleven analytes with the smallest q-values $\left(<3.5 \times 10^{-7}\right)$. Protein concentrations (expressed as RFU values) as a function of renal clearance for the eleven best biomarkers of late-stage (red circles) vs. early-stage CKD (blue circles). doi:10.1371/journal.pone.0015004.g012 
Table 4. Top 11 Potential CKD Biomarkers ${ }^{\S}$.

\begin{tabular}{llll}
\hline Target Protein & p-value & q-value & Mol. Mass (kDa) \\
\hline$\beta_{2}$-Microglobulin & $1.2 \times 10^{-9}$ & $8.0 \times 10^{-8}$ & 11.7 \\
FSTL3 & $1.2 \times 10^{-9}$ & $8.0 \times 10^{-8}$ & 25.0 \\
Pleotrophin & $1.2 \times 10^{-9}$ & $8.0 \times 10^{-8}$ & 15.3 \\
TNF sR-I ${ }^{*}$ & $1.2 \times 10^{-9}$ & $8.0 \times 10^{-8}$ & 21.2 \\
Factor D & $4.8 \times 10^{-9}$ & $2.1 \times 10^{-7}$ & 24.4 \\
IL-15 R $\alpha^{*}{ }^{\dagger}$ & $4.8 \times 10^{-9}$ & $2.1 \times 10^{-7}$ & 25.0 \\
MMP-7 & $8.4 \times 10^{-9}$ & $3.2 \times 10^{-7}$ & 19.1 \\
Angiopoietin-2 & $1.4 \times 10^{-8}$ & $3.5 \times 10^{-7}$ & 54.9 \\
Cystatin C & $1.4 \times 10^{-8}$ & $3.5 \times 10^{-7}$ & 13.3 \\
HCC-1 & $1.4 \times 10^{-8}$ & $3.5 \times 10^{-7}$ & 8.7 \\
URB ${ }^{*}$ & $1.4 \times 10^{-8}$ & $3.5 \times 10^{-7}$ & 105.7 \\
\hline
\end{tabular}

${ }^{\S}$ Based on q-value ranking.

"Smaller isoforms also exist. For example, URB has a $10.3 \mathrm{kDa}$ isoform

* Extracellular domain comprising amino acids 22-211.

'Extracellular domain is $18.4 \mathrm{kDa}$.

doi:10.1371/journal.pone.0015004.t004

to $50 \mu \mathrm{L}$ double-stranded QPCR product and incubated at $25^{\circ} \mathrm{C}$ for 5 minutes with mixing. The beads were washed once with SB1T, and the "sense" strand was eluted from the beads by adding $200 \mu \mathrm{L} 20 \mathrm{mM} \mathrm{NaOH}$ and incubating at $37^{\circ} \mathrm{C}$ for 1 minute with mixing. The eluted strand was discarded and the beads were washed 3 times with SB1T and once with $16 \mathrm{mM}$ $\mathrm{NaCl}$.

SOMAmer sense strand was prepared with the appropriate nucleotide composition by primer extension from the immobilized antisense strand. The beads were resuspended in $20 \mu \mathrm{L}$ primer extension reaction mix (1X KOD DNA Polymerase Buffer, $1.5 \mathrm{mM} \mathrm{MgCl}_{2}, 5 \mu \mathrm{M}$ forward PCR primer, $0.125 \mathrm{U} / \mu \mathrm{L} \mathrm{KOD}$ DNA Polymerase, $0.5 \mathrm{mM}$ each dATP, MedCTP, dGTP, and either dTTP or dUTP analog) and incubated at $68^{\circ} \mathrm{C}$ for 30 minutes with mixing. The beads were washed 3 times with SB1T, and the SOMAmer strand was eluted from the beads by adding $85 \mu \mathrm{L} 20 \mathrm{mM} \mathrm{NaOH}$ and incubating at $37^{\circ} \mathrm{C}$ for 1 minute with mixing. $80 \mu \mathrm{L}$ SOMAmer eluate was transferred to a new tube after magnetic separation, neutralized with $20 \mu \mathrm{L} 80 \mathrm{mM} \mathrm{HCl}$, and buffered with $5 \mu \mathrm{L} 0.1 \mathrm{M}$ HEPES, $\mathrm{pH}$ 7.5.

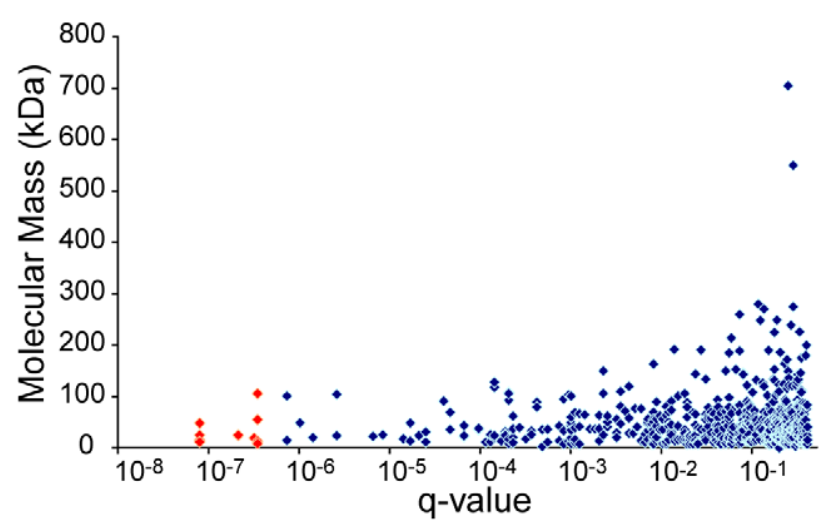

Figure 13. Comparison of a protein's molecular mass and the probability that it is a CKD biomarker (q-value ( $p$-value corrected for false discovery rate)).

doi:10.1371/journal.pone.0015004.g013
Selection Strategy and Feedback. The relative target protein concentration of the selection step was lowered each round in response to the $\mathrm{S} / \mathrm{B}$ ratio as follows (Eq. 1):

$$
\begin{aligned}
& \text { if } \mathrm{S} / \mathrm{B}<10,[\mathrm{P}](\mathrm{i}+1)=[\mathrm{P}] \mathrm{i} \\
& \text { if } 10<\mathrm{S} / \mathrm{B}<100,[\mathrm{P}](\mathrm{i}+1)=[\mathrm{P}] \mathrm{i} / 3.2 \\
& \text { if } \mathrm{S} / \mathrm{B}>100,[\mathrm{P}](\mathrm{i}+1)=[\mathrm{P}] \mathrm{i} / 10
\end{aligned}
$$

where $[\mathrm{P}]=$ protein concentration and $\mathrm{i}=$ current round number. Target protein concentration was lowered by adjusting the mass of target protein beads (and (His) 6 beads) added to the selection step. After each selection round, the convergence state of the enriched DNA mixture was determined. $5 \mu \mathrm{L}$ double-stranded QPCR product was diluted to $200 \mu \mathrm{L}$ with $4 \mathrm{mM} \mathrm{MgCl}_{2}$ containing IX SYBR Green I. Samples were overlaid with $75 \mu \mathrm{L}$ silicone oil and analyzed for convergence as follows.

Nucleic Acid Reassociation Kinetics (G0t) Assay. The sample was thermal cycled with the following protocol: 3 cycles of $98^{\circ} \mathrm{C}, 1$ minute, $85^{\circ} \mathrm{C}, 1$ minute; 1 cycle of $93^{\circ} \mathrm{C}, 1$ minute, $85^{\circ} \mathrm{C}$, 15 minutes. During the 15 minutes at $85^{\circ} \mathrm{C}$, fluorescent images were measured at 5-second intervals. The fluorescence intensity was plotted as a function of log (time) to evaluate the diversity of the sequences.

Measurement of Equilibrium Binding Constants. The equilibrium binding constants of the enriched libraries were measured using MyOne TALON bead partitioning. Radiolabled DNA was renatured by heating to $95^{\circ} \mathrm{C}$ for 3 minutes in SB1T and slowly cooling to $37^{\circ} \mathrm{C}$. Complexes were formed by mixing a low concentration of DNA $\left(\sim 1 \times 10^{-11} \mathrm{M}\right)$ with a range of concentrations of target protein $\left(1 \times 10^{-7} \mathrm{M}\right.$ to $1 \times 10-12 \mathrm{M}$ final $)$ in SB1T and incubating at $37^{\circ} \mathrm{C}$. One-twelfth of each reaction was transferred to a nylon membrane and dried to determine total counts in each reaction. $25 \mu \mathrm{g}$ of MyOne TALON beads was added to the remainder of each reaction and mixed at $37^{\circ} \mathrm{C}$ for one minute. Two-thirds of the reaction was then passed through a MultiScreen HV Plate (Millipore) under vacuum to separate protein-bound complexes from unbound DNA and washed with $100 \mu \mathrm{L} \mathrm{SB1T}$. The nylon membrane and MultiScreen HV Plates were phosphorimaged and the amount of radioactivity in each sample quantified using a FUJI FLA-3000 (Fujifilm Medical Systems). The fraction of captured DNA was plotted as a function of protein concentration and a non-linear curve-fitting algorithm was used to extract equilibrium binding constants ( $\mathrm{K}_{\mathrm{d}}$ values) from the data.

Measurement of Dissociation Rate Constants. The rate constant for SOMAmer:protein complex dissociation was determined for each aptamer by measuring the fraction of preformed aptamer:protein complexes that remain bound after addition of a competitor as a function of time. Radiolabled SOMAmer was renatured as described above. Approximately $5 \times 10-11 \mathrm{M}$ SOMAmer was equilibrated in SB18T $(40 \mathrm{mM}$ HEPES, $100 \mathrm{mM} \mathrm{NaCl}, 5 \mathrm{mM} \mathrm{KCl}, 5 \mathrm{mM} \mathrm{MgCl}_{2}, 0.05 \%$ Tween-20 at pH 7.5) at $37^{\circ} \mathrm{C}$ with protein at a concentration $10 \mathrm{X}$ greater than the measured $\mathrm{K}_{\mathrm{d}}$ value. Samples were then diluted 2X with $40 \mathrm{nM}$ non-labeled SOMAmer or $0.3 \mathrm{mM}$ dextran sulfate in SB18T at various time points. Complexes were partitioned to separate free aptamer from protein:aptamer complexes. The type of partitioning was dependent upon the protein used since not all proteins bind to the same type of partitioning resin. For LBP and Histone H1.2, Zorbax PSM-300A (Agilent Technologies) resin was used for partitioning; for Kallistatin, MyOne TALON beads were used; for biotinylated- 
TIG2, MyOne Streptavidin beads were used. Complexes were captured on the appropriate resin, and the sample was passed through a MultiScreen HV Plate under vacuum. The samples were washed with SB18T. The MultiScreen HV Plates were phosphorimaged and the amount of radioactivity in each sample quantified using a FUJI FLA-3000. The fraction of complex remaining was plotted as a function of time, and the dissociation rate constant was determined by fitting the data to an analytic expression for bimolecular dissociation kinetics using non-linear regression.

Affinity Capture Assay. Not all complexes are able to be captured in the "Catch-3" step of the affinity capture assay (Figure 5), and therefore analyzed by PAGE, because the 3 ' end of the SOMAmer is sometimes involved in its structure or interaction with the target. Additional affinity capture examples for the subset of the CKD-related targets whose complexes can be captured on "Catch-3" beads are shown in Figure 5.

$50 \%$ plasma samples were prepared by diluting ethylene diamine tetraacetic acid (EDTA)-plasma $2 \mathrm{X}$ in SB18T with $2 \mu \mathrm{M}$ Z-Block_2 (the modified nucleotide sequence (AC-BnBn)7AC). The plasma spike samples were prepared by diluting $500 \mathrm{ng}$ protein with the $50 \%$ plasma in SB17T (SB18T with $1 \mathrm{mM}$ EDTA) with 4-(2-Aminoethyl) benzenesulfonyl fluoride hydrochloride (AEBSF) and ethylene glycol tetraacetic acid (EGTA). The plasma samples were prepared by diluting the $50 \%$ plasma in SB17T with AEBSF and EGTA. The buffer spike samples were prepared by diluting $500 \mathrm{ng}$ protein in SB17T with AEBSF and EGTA. These samples were combined with 10 pmoles of SOMAmer to give final concentrations of $10 \%$ plasma, $2 \mathrm{mM}$ AEBSF, $0.5 \mathrm{mM}$ EGTA, and $100 \mathrm{nM}$ SOMAmer. Complexes were formed by incubating at $37^{\circ} \mathrm{C}$ for 45 minutes. $50 \mu \mathrm{L}$ of a $20 \%$ slurry of Streptavidin agarose beads (ThermoFisher Scientific) was added to each sample and shaken for 10 minutes at room temperature. The samples were added to a MultiScreen HV Plate to perform washes under vacuum filtration. Each sample was washed one time quickly with $200 \mu \mathrm{L}$ of SB17T, one time for one minute with $200 \mu \mathrm{L}$ of $100 \mu \mathrm{M}$ biotin in SB17T with shaking, one time with $200 \mu \mathrm{L}$ of SB17T for one minute with shaking, and one time with $200 \mu \mathrm{L}$ of SB17T for nine minutes with shaking. Proteins in the sample were labeled with both biotin and a fluorophore by incubating each sample in $100 \mu \mathrm{L}$ of $1 \mathrm{mM} \mathrm{EZ}$ Link NHS-PEO4-biotin (Pierce), 0.25 mM NHS-Alexa-647 (Invitrogen) in SB17T for five minutes with shaking. Each sample was washed one time with $200 \mu \mathrm{L}$ of $20 \mathrm{mM}$ glycine in SB17T and five times with $200 \mu \mathrm{L}$ of SB17T, shaking each wash for one minute. The final wash was removed using centrifugation at 1000 relative centrifugal force (RCF) for 30 seconds. The beads were resuspended with $100 \mu \mathrm{L}$ of SB17T. SOMAmers (complexed and free) were released from the beads by exposure under a BlackRay light source (UVP XX-Series Bench Lamps, $365 \mathrm{~nm}$ ) for ten minutes with shaking. The samples were spun out of the plate by centrifugation at $1000 \mathrm{RCF}$ for 30 seconds. $10 \mu \mathrm{L}$ of each sample was removed and reserved as "Catch-1 eluate" for SDS-PAGE analysis. The remainder of the samples was captured through the biotinylated proteins by adding $20 \mu \mathrm{L}$ of a $20 \%$ slurry of monomeric Avidin beads and shaking for ten minutes. The beads were transferred to a MultiScreen HV Plate and washed four times with $100 \mu \mathrm{L}$ of SB17T for one minute with shaking. The final wash was removed using centrifugation at $1000 \mathrm{RCF}$ for 30 seconds. Proteins were eluted from the beads by incubating each sample with $100 \mu \mathrm{L}$ of $2 \mathrm{mM}$ biotin in SB17T for five minutes with shaking. Each eluate was transferred to $0.4 \mathrm{mg}$ MyOne Streptavidin beads with a bound biotinylated-primer complementary to the $3^{\prime}$ fixed region of the SOMAmer. The samples were incubated for five minutes with shaking to anneal the bead-bound fixed region to the SOMAmer complexes. Each sample was washed two times with $100 \mu \mathrm{L}$ of $1 \mathrm{XSB} 17 \mathrm{~T}$ for one minute each with shaking and one time with $100 \mu \mathrm{L}$ of 1XSB19T $(5 \mathrm{mM}$ HEPES, $100 \mathrm{mM}$ NaCl, $5 \mathrm{mM} \mathrm{KCl,} 5 \mathrm{mM} \mathrm{MgCl}_{2}, 1 \mathrm{mM}$ EDTA, 0.05\% Tween-20, pH 7.5) for one minute with shaking, all by magnetic separation. The complexes were eluted by incubating with $45 \mu \mathrm{L}$ of $20 \mathrm{mM} \mathrm{NaOH}$ for two minutes with shaking. $40 \mu \mathrm{L}$ of each eluate was added to $10 \mu \mathrm{L}$ of $80 \mathrm{mM} \mathrm{HCl}$ with $0.05 \%$ Tween-20 in a new plate. $10 \mu \mathrm{L}$ of each sample was removed and reserved as "Catch-2 aptamer-bound eluate" for SDS-PAGE analysis. Gel samples were run on NuPAGE $4-12 \%$ Bis Tris Glycine gels (Invitrogen) under reducing and denaturing conditions according to the manufacturer's directions. Gels were imaged on an Alpha Innotech FluorChem Q scanner in the Cy5 channel to image the proteins.

\section{LC-MS/MS Protein Identification}

Tandem mass spectra were collected at NextGen Sciences. Scaffold (Proteome Software) was used to probabilistically validate protein identifications derived from MS/MS sequencing results using the X!Tandem [43] ProteinProphet computer algorithms [44].

Database searching. All tandem MS/MS samples were analyzed using Mascot (Matrix Science; version Mascot). Mascot was set up to search the ipi.HUMAN.v3.53_plusREV database (147698 entries) assuming the digestion enzyme trypsin. Mascot was searched with a fragment ion mass tolerance of $0.50 \mathrm{Da}$ and a parent ion tolerance of 5.0 PPM. Iodoacetamide derivative of cysteine was specified in Mascot as a fixed modification. Scarbamoylmethylcysteine cyclization (N-terminus) of the nterminus, deamidation of asparagine, oxidation of methionine and acetylation of the n-terminus were specified in Mascot as variable modifications.

Criteria for protein identification. Scaffold (version Scaffold_2_04_01, Proteome Software Inc) was used to validate MS/MS based peptide and protein identifications. Peptide identifications were accepted if they could be established at greater than $50.0 \%$ probability as specified by the Peptide Prophet algorithm [45]. Protein identifications were accepted if they could be established at greater than $90.0 \%$ probability and contained at least 2 identified peptides. Protein probabilities were assigned by the Protein Prophet algorithm [44]. Proteins that contained similar peptides and could not be differentiated based on MS/MS analysis alone were grouped to satisfy the principles of parsimony.

\section{Proteomic Affinity Assay Method}

All steps of the proteomic affinity assay were performed at room temperature unless otherwise indicated.

Sample thawing and plating. Aliquots of $100 \%$ serum or EDTA- plasma, stored at $-80^{\circ} \mathrm{C}$, were thawed by incubating in a $25^{\circ} \mathrm{C}$ water bath for ten minutes. After thawing the samples were stored on ice during mixing and prior to sample dilution. Samples were mixed by gentle vortexing (setting \# 4 on Vortex Genie, Scientific Industries) for 8 seconds. A 20\% sample solution was prepared by transferring $16 \mu \mathrm{L}$ of thawed sample into 96-well plates (Hybaid Omnitube $0.3 \mathrm{~mL}$, ThermoFisher Scientific) containing $64 \mu \mathrm{L}$ per well of the appropriate sample diluent at $4^{\circ} \mathrm{C}$. Sample diluent for serum was $0.8 \mathrm{x}$ SB 17 with $0.6 \mathrm{mM}$ $\mathrm{MgCl}_{2}, 2$ mM EGTA, $2 \mu \mathrm{M}$ Z-Block_2, 0.05\% Tween and for EDTA-plasma was $0.8 \mathrm{x} \mathrm{SB} 18$ with $0.8 \mathrm{mM} \mathrm{MgCl}_{2}, 2 \mathrm{mM}$ EGTA, $2 \mu \mathrm{M}$ Z-Block_2, $0.05 \%$ Tween. This plate was stored on ice until the next sample dilution steps were initiated. 
Preparation of $10 \%, 1 \%$ and $0.03 \%$ SOMAmer Solutions. SOMAmers were grouped into three unique mixes. The placing of a SOMAmer within a mix was empirically determined by assaying a dilution series of serum or plasma with each SOMAmer and identifying the sample dilution that gave the largest linear range of signal. The segregation of SOMAmers and mixing with different dilutions of sample $(10 \%, 1 \%$ or $0.03 \%)$ allow the assay to span a $10^{7}$-fold range of protein concentration. The composition of the custom SOMAmer mixes was slightly different between plasma and serum as expected due to variation in protein composition of these two media. The custom stock SOMAmer solutions for $10 \%, 1 \%$ and $0.03 \%$ serum and plasma were prepared and stored at 8x concentration in SB17T.

For each assay run, the three 8x SOMAmer solutions were diluted separately 1:4 into SB17T to achieve $2 \mathrm{x}$ concentration. Each diluted SOMAmer master mix was heated to $95^{\circ} \mathrm{C}$ for five minutes and then to $37^{\circ} \mathrm{C}$ for 15 minutes. $55 \mu \mathrm{L}$ of each $2 \mathrm{x}$ SOMAmer mix was manually pipetted into a 96-well plate resulting in three plates with $10 \%, 1 \%$ or $0.03 \%$ SOMAmer mixes. After mixing with sample, the final individual SOMAmer concentration ranged from $0.25-4 \mathrm{nM}$ for serum, $0.5 \mathrm{nM}$ for plasma.

Equilibration. A 2\% sample plate was prepared by diluting the 20\% sample 1:10 into SB17T using the Beckman Coulter Biomek $\mathrm{Fx}^{\mathrm{P}}$ (Beckman Coulter). A $0.06 \%$ sample plate was prepared by diluting the $2 \%$ sample plate $1: 31$ into SB17T. The three sample dilutions were then transferred to their respective SOMAmer solutions by adding $55 \mu \mathrm{L}$ of the sample to $55 \mu \mathrm{L}$ of the appropriate $2 \mathrm{x}$ SOMAmer mix. The plates were sealed with a foil seal (Microseal 'F' Foil, Bio-Rad) and incubated at $37^{\circ} \mathrm{C}$ for 3.5 hours.

Preparation of Catch-1 Bead Plates. $\quad 133.3 \mu \mathrm{L}$ of a $7.5 \%$ Streptavidin-agarose bead slurry in SB17T was added to each well of three pre-washed 0.45 um filter plates. Each well of beads was washed once with $200 \mu \mathrm{L}$ SB17T using vacuum filtration to remove the wash and then resuspended in $200 \mu \mathrm{L} \mathrm{SB17T}$.

Gatch-1 Bead Gapture. All subsequent steps were performed by the Beckman Coulter Biomek $\mathrm{Fx}^{\mathrm{P}}$ robot unless otherwise noted. After the 3.5 hour equilibration, $100 \mu \mathrm{L}$ of the $10 \%, 1 \%$ and $0.03 \%$ equilibration binding reactions was transferred to their respective Catch-1 Streptavidin agarose filter plates and incubated with shaking for ten minutes. Unbound solution was removed via vacuum filtration. Each set of Catch-1 beads was washed with $190 \mu \mathrm{L}$ of $100 \mu \mathrm{M}$ biotin in SB17T and then $190 \mu \mathrm{L}$ of $\mathrm{SB} 17 \mathrm{~T}$ using vacuum filtration to remove the wash. $190 \mu \mathrm{L}$ SB17T was added to each well in the Catch-1 plates and incubated with shaking for ten minutes at $25^{\circ} \mathrm{C}$. The wash was removed via vacuum filtration and the bottom of the filter plates blotted to remove droplets using the on-deck blot station.

Biotinylation of Proteins. An aliquot of $100 \mathrm{mM}$ NHSPEO4-biotin in DMSO was thawed at $37^{\circ} \mathrm{C}$ for six minutes and diluted to $1 \mathrm{mM}$ with $\mathrm{SB} 17 \mathrm{~T}$ at $\mathrm{pH} 7.25$. $100 \mu \mathrm{L}$ of the NHSPEO4-biotin was added to each well of each Catch-1 filter plate and incubated with shaking for five minutes. Each biotinylation reaction was quenched by adding $150 \mu \mathrm{L}$ of $20 \mathrm{mM}$ glycine in SB17T to the Catch-1 plates with the NHS-PEO4-biotin. Plates were incubated for one minute with shaking, vacuum filtrated, and $190 \mu \mathrm{L} 20 \mathrm{mM}$ glycine SB17T was added to each well in the plate. The plates were incubated for one minute, shaking before removal by vacuum filtration. $190 \mu \mathrm{L}$ of SB17T was added to each well and removed by vacuum filtration. The wells of the Catch-1 plates were subsequently washed three times by adding $190 \mu \mathrm{L} \mathrm{SB17T}$, incubating for one minute with shaking followed by vacuum filtration. After the last wash the plates were centrifuged at $1000 \mathrm{rpm}$ for one minute over a $1 \mathrm{~mL}$ deep-well plate to remove extraneous volume before elution. Centrifugation was performed off deck.

Kinetic Challenge and Photo-Cleavage. $85 \mu \mathrm{L}$ of $10 \mathrm{mM}$ dextran sulfate in SB17T was added to each well of the filter plates. The filter plates were placed onto a Thermal Shaker (Eppendorf) under a BlackRay light source and irradiated for ten minutes with shaking. The photo-cleaved solutions were sequentially eluted from each Catch-1 plate into a common deep well plate by centrifugation at $1000 \mathrm{rpm}$ for one minute each.

Gatch-2 Bead Gapture. In bulk, MyOne-Streptavidin C1 beads were washed two times for 5 minutes each with equal volume of $20 \mathrm{mM} \mathrm{NaOH}$ and three times with an equal volume of $\mathrm{SB} 17 \mathrm{~T}$. Beads were resuspended in SB17T to a concentration of $10 \mathrm{mg} / \mathrm{mL}$. After resuspension, $50 \mu \mathrm{L}$ of this solution was manually pipetted into each well of a 96-well plate and stored at $4^{\circ} \mathrm{C}$ until Catch-2. During Catch-2, the wash supernatant was removed via magnetic separation. All of the photo-cleaved eluate was pipetted onto the MyOne magnetic beads and incubated with shaking at $25^{\circ} \mathrm{C}$ for five minutes. The supernatant was removed from the MyOne beads via magnetic separation and $75 \mu \mathrm{L}$ of $\mathrm{SB} 17 \mathrm{~T}$ was transferred to each well. The plate was mixed for one minute at $37^{\circ} \mathrm{C}$ with shaking and then $75 \mu \mathrm{L}$ of $60 \%$ glycerol (in $\mathrm{SB} 17 \mathrm{~T}$ ) at $37^{\circ} \mathrm{C}$ was transferred to each well. The plate was mixed for another minute at $37^{\circ} \mathrm{C}$ with shaking. The wash was removed via magnetic separation. These washes were repeated two more times. After removal of the third glycerol wash from the MyOne beads, $150 \mu \mathrm{L}$ of SB17T was added to each well and the plates incubated at $37^{\circ} \mathrm{C}$ with shaking for one minute before removal by magnetic separation. The MyOne beads were washed a final time using $150 \mu \mathrm{L} \mathrm{SB19T}$ with incubation for one minute, prior to magnetic separation.

Gatch-2 Bead Elution and Neutralization. SOMAmers were eluted from MyOne beads by incubating each well of beads with $105 \mu \mathrm{L}$ of $100 \mathrm{mM}$ CAPSO $\mathrm{pH} 10,1 \mathrm{M} \mathrm{NaCl}, 0.05 \%$ Tween with shaking for five minutes. $90 \mu \mathrm{L}$ of each eluate was transferred during magnetic separation to a new 96-well plate containing $10 \mu \mathrm{L}$ of $500 \mathrm{mM} \mathrm{HCl}, 500 \mathrm{mM}$ HEPES, $0.05 \%$ Tween-20, pH 7.5.

Hybridization. $20 \mu \mathrm{L}$ of each neutralized Catch-2 eluate was transferred to a new 96-well plate and $5 \mu \mathrm{L}$ of $10 \mathrm{x}$ Agilent Block (Oligo aCGH/ChIP-on-chip Hybridization Kit, Large Volume, Agilent Technologies 5188-5380), containing a 10x spike of hybridization controls (10 Cy3 SOMAmers) was added to each well. After removing the plate from the robot, $25 \mu \mathrm{L}$ of $2 \mathrm{x}$ Agilent Hybridization buffer (Oligo aCGH/ChIP-on-chip Hybridization Kit, Agilent Technologies) was manually pipetted to the each well of the plate containing the neutralized samples and blocking buffer. $40 \mu \mathrm{L}$ of this solution was manually pipetted into each "well" of the hybridization gasket slide (Hybridization Gasket Slide - 8 microarrays per slide format, Agilent Technologies). Custom Agilent microarray slides containing 10 probes per array complementary to 40 nucleotide selected region of each SOMAmer with a 20x dT linker were placed onto the gasket slides according to the manufacturer's protocol. Each assembly (Hybridization Chamber Kit - SureHyb enabled, Agilent Technologies) was tightly clamped and loaded into a hybridization oven for 19 hours at $60^{\circ} \mathrm{C}$ rotating at $20 \mathrm{rpm}$.

Post-Hybridization Washing. Approximately $400 \mathrm{~mL}$ Wash Buffer 1 (Oligo aCGH/ChIP-on-chip Wash Buffer 1, Agilent Technologies) was placed into each of two separate glass staining dishes. Six of the twelve slide/gasket assemblies were sequentially disassembled into the first staining dish containing 
Wash Buffer 1. Once disassembled, the slide was quickly transferred into a slide rack in a second staining dish containing Wash Buffer 1. The slides were incubated for five minutes in Wash Buffer 1 with mixing via magnetic stir bar. The slide rack was then transferred to the $37^{\circ} \mathrm{C}$ Wash Buffer 2 (Oligo aCGH/ChIP-onchip Wash Buffer 2, Agilent Technologies) and allowed to incubate for five minutes with stirring. The slide rack was transferred to a fourth staining dish containing acetonitrile and incubated for five minutes with stirring.

Microarray Imaging. The microarray slides were imaged with a microarray scanner (Agilent G2565CA Microarray Scanner System, Agilent Technologies) in the Cy3-channel at $5 \mu \mathrm{m}$ resolution at $100 \%$ PMT setting and the XRD option enabled at 0.05 . The resulting tiff images were processed using Agilent feature extraction software version 10.5.1.1 with the GE1_105_ Dec08 protocol.

\section{Serum and Plasma Reproducibility Studies}

For each plate, five aliquots of plasma or serum from 18 individuals were thawed and plated as described below. Six wells containing only buffer were run on every plate. Serum and plasma samples were run on separate plates because they require slightly different buffers as indicated above. Three plates of each sample type were run over the course of several days and included using different lots of buffers and other reagents that might be expected to change within a large study.

\section{Limits of Quantification (LOQ) Experiment}

For the LOQ experiments, four different sets of protein mixes were prepared for each of the three SOMAmer mixes, 10\%, $1 \%$ or $0.03 \%$, for a total of 12 mixes and 356 proteins. The proteins for each mix were chosen to avoid combining known protein binding partners and known protease-substrate pairs. The proteins were diluted into SB17T containing $2 \mu \mathrm{M}$ Z-Block_2 so that each protein was at a final concentration of $20 \mathrm{nM}$. The protein solutions were serially diluted 15.8 -fold into SB17T for a total of six points (lowest concentration: $20.3 \mathrm{fM}$ ). All of the protein preparation was maintained on ice. Eight replicate protein titrations per set were pipetted into 96-well plates.

Precision profiles. The coefficient of variation $(\mathrm{CV})$, the standard deviation $(\sigma)$ of the calculated concentration divided by the concentration, is typically determined for computing LOQs. As analyte concentration approaches zero, the assay CV diverges. Similarly, for large analyte concentrations near the assay plateau, small changes in assay signal can give rise to large changes in calculated concentration, leading again to a divergence in $\mathrm{GVs}$. In between these two divergences in $\mathrm{CV}$ s lies a concentration range for which the assay measurements have $\mathrm{CVs}$ of a desired limit or less.

We set this limit at 20\% CV and determined the upper and lower LOQs as those high and low concentrations equal to $20 \%$ CV. Standard curves were computed by averaging the relative fluorescent units (RFUs) for eight replicate measurements at each concentration. A standard four parameter Hill model (Eq. 2) in log transformed RFU was used to fit the dose-response curves, where $\mathrm{x}$ denotes an analyte concentration.

$$
\begin{aligned}
\log R F U= & \left(\log R F U_{\text {plateau }}-\log R F U_{\text {baseline }}\right) \frac{x^{\alpha}}{x^{\alpha}+K^{\alpha}} \\
& +\log R F U_{\text {baseline }}
\end{aligned}
$$

Two distinct approaches were used to compute precision profiles from these data. The first approach modeled the standard deviation for calculated concentrations $\sigma_{x}$, obtained by averaging the eight replicates at each concentration, with a quadratic function from which the precision profile was directly obtained (Figure S3). The second approach is to model the standard deviation of the assay response $\sigma_{\log R F U}$ with a quadratic function and then use the dose-response function to compute the variance in concentration from the response variance. This is not easily accomplished for the dose-response function used here but linearizing the function at a concentration $x$ leads to the following simplification (Eq. 3 and 4).

$$
\sigma_{x}=\frac{\sigma_{\log R F U}}{\left(\frac{\partial \log R F U}{\partial x}\right)}
$$

$$
\left(\frac{\partial \log R F U}{\partial x}\right)=\left(\log R F U_{\text {plateau }}-\log R F U_{\text {baseline }}\right) \frac{\alpha x^{\alpha-1} K^{\alpha}}{\left(x^{\alpha}+K^{\alpha}\right)^{2}}(4)
$$

Typically, the assay $\mathrm{CV}$ in response units $\left(\sigma_{\log R F U} / \log R F U\right)$ is fairly constant so using a quadratic function to model $\sigma_{R F U}$ as a function of concentration should suffice.

We produced the full precision profile for each SOMAmer tested using both numerical approaches outlined above (Figure S3). Both methods give essentially the same result in this case for LLOQ and ULOQ. This particular analyte shows a remarkable five-log quantification range at a $20 \% \mathrm{CV}$ cutoff with an LLOQ of 0.4-0.6 pM and a ULOQ of 40-50 nM. In general there is good agreement between the two different methods for computing precision profiles, and the assay response $\sigma_{\log R F U}$ method was used to calculate the values reported in Table S2.

\section{Chronic Kidney Disease}

GKD serum samples were collected by the Rogosin Institute for the clinical study entitled "Quantification of inflammatory and immune mediators of CKD in patient serum, whole blood and urine: Correlation with CKD disease stage progression". Both the original study and the biomarker study reported here were approved by the Institutional Review Board at Weil Medical College of Cornell University. The clinical study design specified that samples be collected from 25 healthy controls with no renal disease and 25 subjects at each stage of CKD (1-5) for a total of 150 subjects. Our biomarker study included serum samples from 42 subjects that were available at the time this study was conducted. Table 3 summarizes the population demographics. The groups are well matched by gender, ethnicity, age, weight, and body mass index. Renal function, measured by the estimated glomerular filtration rate (eGFR, calculated with the MDRD formula for creatinine clearance [46]), is substantially different in the two groups (Table 3).

\section{Clinical Data Processing}

Assay Normalization. Assay normalization was performed to reduce signal variation potentially introduced during the assay. Each sample in a study was normalized using a set of SOMAmers that have the lowest overall relative signal variation across all samples within a study. For each normalization SOMAmer, its median value was calculated from all samples in the study, and together these median values were used to calculate a scaling factor for each individual sample. The scaling factor was the mean of a series of values, one for each normalization SOMAmer, calculated as the sample signal divided by the median signal for the study. When 
applied to a sample, this procedure brings the signals corresponding to SOMAmers in the normalization set closer to the median values across the assay, and reduces the observed variation between replicate samples for all SOMAmers. Because the assay splits each clinical sample into three dilutions, assay normalization was performed separately on the three SOMAmer groups corresponding to the $10 \%, 1 \%$, and $0.1 \%$ dilutions. Dilution normalization applies the same constant factor to every signal in that dilution from any given sample. This factor varied between samples in the range from 0.8 to 1.2 , and was typically within $10 \%$ of unity.

Between-run Galibration. To compare samples between assay runs with slightly different conditions, we have applied calibration to the individual SOMAmers signals. For this we apply a multiplicative correction factor specific to each SOMAmer, but invariant with respect to the sample (in contrast to normalization in which the factor was specific to the sample and did not vary from SOMAmer to SOMAmer within a sample). To calculate the calibration constant for each SOMAmer, we measure a set of eight calibrator samples derived from blood from the same individual in each sample set. From these calibrator sample measurements, we can standardize the signals from a sample within one run by applying the calibration coefficient for each SOMAmer that scales the median calibrator signal of that aptamer to a reference standard for that aptamer.

\section{Supporting Information}

Figure S1 Reproducibility of measurements in plasma and serum. The cumulative distribution function (cdf) of intrarun coefficients of variation $(\mathrm{CVs})$ and inter-run $\mathrm{CVs}$ for plasma and serum are shown for the three dilutions mixes: 10\% (red), $1 \%$ (green), and $0.03 \%$ (blue).

(TIF)

Figure S2 Precision profile for a2-Antiplasmin. A. Representative dose-response curve calculated with a fourparameter fit to average concentration (blue circles) of eight replicate protein measurements (red circles). B. Standard deviation $\sigma_{\mathrm{x}}$ of calculated concentration (blue circles) with quadratic fit (solid line) and 95\% confidence (dashed lines). C. Standard deviation of assay response shown as $\sigma_{\operatorname{logRFU}}$ (red circles) with quadratic fit (solid line) and the 95\% confidence (dashed lines). D. Precision profiles for assay response computed by modeling $\sigma_{\mathrm{x}}$ (blue) and $\sigma_{\operatorname{logRFU}}(\mathrm{red})$.

(TIF)

Figure S3 Gumulative probability functions (cdfs) for limits of quantification computed from precision for 356

\section{References}

1. Zichi D, Eaton B, Singer B, Gold L (2008) Proteomics and diagnostics: Let's Get Specific, again. Curr Opin Chem Biol 12: 78-85.

2. Pan S, Aebersold R, Chen R, Rush J, Goodlett DR, et al. (2009) Mass spectrometry based targeted protein quantification: methods and applications. J Proteome Res 8: 787-797.

3. Service RF (2008) Proteomics. Proteomics ponders prime time. Science 321: 1758-1761.

4. Liotta L, Petricoin F (2010) Mass Spectrometry-Based Protein Biomarker Discovery and Measurement: Sensitivity is the Greatest Hurdle. Clinical Proteomics 6: 4-5.

5. Silberring J, Ciborowski P (2010) Biomarker discovery and clinical proteomics. Trends Analyt Chem 29: 128

6. Mitchell P (2010) Proteomics retrenches. Nat Biotechnol 28: 665-670.

7. Bell AW, Deutsch EW, Au CE, Kearney RE, Beavis R, et al. (2009) A HUPO test sample study reveals common problems in mass spectrometry-based proteomics. Nat Methods.

8. Aebersold R (2009) A stress test for mass spectrometry-based proteomics. Nat Methods 6: 411-412 analytes measured in buffer. A. Distribution of LLOQs; median $0.9 \mathrm{pM}$; inter-quartile range $0.3 \mathrm{pM}-3.9 \mathrm{pM}$; lowest $10 \mathrm{fM}$. B. Distribution of ULOQs; median $1.5 \mathrm{nM}$; inter-quartile range $0.7 \mathrm{nM}-4.5 \mathrm{nM}$. C. Distribution of $\log$ ROQ; median quantification range $\sim 3$ logs.

(TIF)

Figure S4 Comparison of LLOQ, ULOQ, and ROQ for 28 analytes measured in buffer and plasma. All data were computed by modeling $\sigma_{\log R F U}$.

(TIF)

Table S1 List of the 813 proteins measured in the current version of the assay and the subset of 614 proteins measured in the CKD study.

(DOC)

Table S2 List of limits of quantification for 356 representative proteins measured in buffer.

(DOG)

Table S3 List of measured limits of quantification for proteins spiked into buffer and plasma.

(DOC)

Table S4 List of 60 proteins identified that varied between early and late stage CKD with a q-value of $4.2 \times 10^{-4}$.

(DOC)

\section{Acknowledgments}

We thank all our colleagues in the genesis and evolution of aptamer science and developing aptamer-based biotechnology over the past decades. They contributed immeasurably with countless insights about aptamers and the nature of biology. We especially thank past and present colleagues at the University of Colorado, NeXstar Pharmaceuticals, and SomaLogic. We also thank the following individuals for providing proteins: Fanqing Chen, Lawrence Berkeley National Labs; Brian Fox, University of Wisconsin; Michael Gelb, University of Washington; Tsuotmu Ohta, National Cancer Center of Japan; Yves Pommier and Christoph Rader, National Cancer Institute of the United States; Dylan Taatjes, University of Colorado.

\section{Author Contributions}

All authors contributed extensively to the work presented in this paper. Provided CKD samples and critically evaluated results: DL TP. Wrote the manuscript with input from the entire team: NJ JJW SKW DZ.

9. Addona TA, Abbatiello SE, Schilling B, Skates SJ, Mani DR, et al. (2009) Multisite assessment of the precision and reproducibility of multiple reaction monitoringbased measurements of proteins in plasma. Nat Biotechnol 27: 633-641.

10. Fredriksson S, Dixon W, Ji H, Koong AC, Mindrinos M, et al. (2007) Multiplexed protein detection by proximity ligation for cancer biomarker validation. Nat Methods 4: 327-329.

11. Schweitzer B, Roberts S, Grimwade B, Shao W, Wang M, et al. (2002) Multiplexed protein profiling on microarrays by rolling-circle amplification. Nat Biotechnol 20: 359-365.

12. Borrebaeck C, Wingren C (2007) High-throughput proteomics using antibody microarrays: an update. Expert Rev Mol Diagn 7: 673-686.

13. Ellington AD, Szostak JW (1990) In vitro selection of RNA molecules that bind specific ligands. Nature 346: 818-822.

14. Tuerk C, Gold L (1990) Systematic evolution of ligands by exponential enrichment: RNA ligands to bacteriophage T4 DNA polymerase. Science 249: 505-510.

15. Gragoudas ES, Adamis AP, Cunningham ET, Jr., Feinsod M, Guyer DR (2004) Pegaptanib for neovascular age-related macular degeneration. N Engl J Med 351: 2805-2816. 
16. Tarasow TM, Tarasow SL, Eaton BE (1997) RNA-catalysed carbon-carbon bond formation. Nature 389: 54-57.

17. Brody EN, Gold L (2000) Aptamers as therapeutic and diagnostic agents. J Biotechnol 74: 5-13.

18. Famulok M, Hartig JS, Mayer G (2007) Functional aptamers and aptazymes in biotechnology, diagnostics, and therapy. Chem Rev 107: 3715-3743.

19. Gold L (1995) Oligonucleotides as Research, Diagnostic, and Therapeutic Agents. J Biol Chem 270: 13581-13584.

20. Binz HK, Amstutz P, Pluckthun A (2005) Engineering novel binding proteins from nonimmunoglobulin domains. Nat Biotechnol 23: 1257-1268.

21. Eaton BE (1997) The joys of in vitro selection: chemically dressing oligonucleotides to satiate protein targets. Curr Opin Chem Biol 1: 10-16.

22. Dewey T, Mundt A, Crouch G, Zyniewski M, Eaton B (1995) New Uridine Derivatives for Systematic Evolution of RNA Ligands by Exponential Enrichment. J Am Chem Soc 117: 8474-8475.

23. Gugliotti LA, Feldheim DL, Eaton BE (2004) RNA-mediated metal-metal bond formation in the synthesis of hexagonal palladium nanoparticles. Science 304: 850-852.

24. Vaught JD, Bock C, Carter J, Fitzwater T, Otis M, et al. (2010) Expanding the chemistry of DNA for in vitro selection. J Am Chem Soc 132: 4141-4151.

25. Hopfield JJ (1974) Kinetic proofreading: a new mechanism for reducing errors in biosynthetic processes requiring high specificity. Proc Natl Acad Sci USA 71: 4135-4139.

26. Ninio J (1975) Kinetic amplification of enzyme discrimination. Biochimie 57: 587-595.

27. Vaught JD, Dewey T, Eaton BE (2004) T7 RNA polymerase transcription with 5-position modified UTP derivatives. J Am Chem Soc 126: 11231-11237.

28. Levey AS, Atkins R, Coresh J, Cohen EP, Collins AJ, et al. (2007) Chronic kidney disease as a global public health problem: approaches and initiatives - a position statement from Kidney Disease Improving Global Outcomes. Kidney Int 72: 247-259.

29. Chaudhary K, Phadke G, Nistala R, Weidmeyer CE, McFarlane SI, et al. (2010) The emerging role of biomarkers in diabetic and hypertensive chronic kidney disease. Curr Diab Rep 10: 37-42.

30. Giannelli SV, Patel KV, Windham BG, Pizzarelli F, Ferrucci L, et al. (2007) Magnitude of underascertainment of impaired kidney function in older adults with normal serum creatinine. J Am Geriatr Soc 55: 816-823.

31. Nickolas TL, Barasch J, Devarajan P (2008) Biomarkers in acute and chronic kidney disease. Curr Opin Nephrol Hypertens 17: 127-132.

32. Venturoli D, Rippe B (2005) Ficoll and dextran vs. globular proteins as probes for testing glomerular permselectivity: effects of molecular size, shape, charge, and deformability. Am J Physiol Renal Physiol 288: F605-613.
33. Stevens LA, Coresh J, Greene T, Levey AS (2006) Assessing kidney functionmeasured and estimated glomerular filtration rate. N Engl J Med 354: 2473-2483.

34. van Riemsdijk-van Overbeeke IC, Baan CC, Hesse CJ, Loonen EH, Niesters HG, et al. (2000) TNF-alpha: mRNA, plasma protein levels and soluble receptors in patients on chronic hemodialysis, on CAPD and with endstage renal failure. Clin Nephrol 53: 115-123.

35. Pascual M, Steiger G, Estreicher J, Macon K, Volanakis JE, et al. (1988) Metabolism of complement factor D in renal failure. Kidney Int 34: 529-536.

36. Vanholder R, Van Laecke S, Glorieux G (2008) The middle-molecule hypothesis 30 years after: lost and rediscovered in the universe of uremic toxicity? J Nephrol 21: 146-160.

37. Doggrell SA (2005) Pegaptanib: the first antiangiogenic agent approved for neovascular macular degeneration. Expert Opin Pharmaco 6: 1421-1423.

38. Ruckman J, Green LS, Beeson J, Waugh S, Gillette WL, et al. (1998) 2'Fluoropyrimidine RNA-based aptamers to the 165-amino acid form of vascular endothelial growth factor (VEGF165). Inhibition of receptor binding and VEGF-induced vascular permeability through interactions requiring the exon 7encoded domain. J Biol Chem 273: 20556-20567.

39. Jenison RD, Gill SC, Pardi A, Polisky B (1994) High-resolution molecular discrimination by RNA. Science 263: 1425-1429.

40. Ostroff RM, Bigbee WL, Franklin W, Gold L, Mehan M, et al. (2010) Unlocking biomarker discovery: Large scale use of aptamer proteomic technology for early detection of lung cancer. PLoS ONE 5(11): e15004. doi: 10.1371/journal.pone.0015003.

41. Schneider D, Nieuwlandt D, Eaton B, Stanton M, Gupta S, et al. (2009) Multiplexed Analyses of Test Samples. US2009/0042206. USA.

42. Zichi D, Wilcox SK, Bock C, Schneider DJ, Eaton B, et al. (2009) Method for Generating Aptamers with Improved Off-Rates. US2009/0004667. USA

43. Craig R, Beavis RC (2003) A method for reducing the time required to match protein sequences with tandem mass spectra. Rapid Commun Mass Spectrom 17: $2310-2316$

44. Nesvizhskii AI, Keller A, Kolker E, Aebersold R (2003) A statistical model for identifying proteins by tandem mass spectrometry. Anal Chem 75: 4646-4658.

45. Keller A, Nesvizhskii AI, Kolker E, Aebersold R (2002) Empirical statistical model to estimate the accuracy of peptide identifications made by MS/MS and database search. Anal Chem 74: 5383-5392.

46. Levey AS, Bosch JP, Lewis JB, Greene T, Rogers N, et al. (1999) A more accurate method to estimate glomerular filtration rate from serum creatinine: a new prediction equation. Modification of Diet in Renal Disease Study Group. Ann Intern Med 130: 461-470. 